\title{
Order and type as measures of growth for convex or entire functions
}

\author{
Christer O. Kiselman
}

Contents:
1. Introduction
2. Order and type in classical complex analysis
3. Relative order and type of convex functions
4. Order and type in duality
5. The infimal convolution
6. The order of an entire function
7. A geometric characterisation of the relative order
8. An extension problem for holomorphic functions
9. Whittaker's decomposition theorem
10. Inequalities for the infimal convolution
11. Inequalities for the infimal convolution, one variable
12. The type of an entire function
References

\section{Introduction}

The notions of order and type of entire functions are classical in complex analysis. They result from a comparison of a given function with standard functions. The purpose of this paper is to generalise this comparison in such a way that order and type become dual to each other in the sense of convex analysis (section 4), and to show that the concept of order so obtained appears as the natural answer to a problem of extrapolation: to extend convex functions from the union of two parallel hyperplanes to as large a set as possible (section 7 ). Then we return to entire functions to consider an analogous extension problem for them (section 8).

It is shown that the relative order of one function with respect to another can always be calculated from the growth of its Taylor coefficients (section 6). This is true for the type only if the growth is sufficiently regular (section 12). We also consider a generalisation of the notion of regular growth of entire functions, and show that Whittaker's decomposition theorem holds for functions which are of irregular growth (section 9).

In [6] I studied order and type from this point of view, using methods from my paper [5]. For earlier developments see the references in that paper. See also Kiselman $[\mathbf{7}, \mathbf{8}]$. A different approach to the relation between maximum modulus and Taylor coefficients is presented in Freund \& Görlich [1].

This work has been partially supported by the Swedish Natural Science Research Council. 
I am grateful to Harvir S. Kasana for discussions concerning Whittaker's decomposition theorem.

\section{Order and type in classical complex analysis}

Let $h$ be an entire function in $\mathbf{C}^{n}$. Its order and type are defined classically by comparing $h$ with the function $\exp \left(b|z|^{a}\right)$ for various choices of the parameters $a$ and $b$. More precisely, one considers first estimates

$$
|h(z)| \leqslant C_{a} e^{|z|^{a}}, \quad z \in \mathbf{C}^{n}
$$

and defines the order $\rho$ as the infimum of all numbers $a$ for which such an estimate holds $(0<a<+\infty ; 0 \leqslant \rho \leqslant+\infty)$. In the case where $0<\rho<+\infty$ one then considers all numbers $b$ such that

$$
|h(z)| \leqslant C_{b} e^{b|z|^{\rho}}, \quad z \in \mathbf{C}^{n}
$$

for some constant $C_{b}$. The type (with respect to the order $\rho$ ) is then the infimum $\sigma$ of all such numbers $b(0<b<+\infty ; 0 \leqslant \sigma \leqslant+\infty)$.

For the order we have the formula

$$
\rho=\operatorname{order}(h)=\limsup _{r \rightarrow+\infty} \sup _{|z|=r} \frac{\log \log |h(z)|}{\log r} .
$$

Now $\sup _{|z|=r} \log |h(z)|$ is a convex function of $\log r$ in view of the Hadamard threecircle-theorem, so it is natural to consider the function

$$
f(t)=\sup _{|z|=e^{t}} \log |h(z)|, \quad t \in \mathbf{R}
$$

we shall call it the growth function of $h$. The definition of order then means that we consider all numbers $a$ such that

$$
f(t) \leqslant e^{a t}+C_{a}, \quad t \in \mathbf{R},
$$

for some constant $C_{a}$, and then define the order as the infimum of all such numbers $a$. (The role of the constant $C_{a}$ is to eliminate all influence of values of $f$ at any particular point.) Similarly, the type (for order $\rho$ ) is the infimum of all numbers $b$ such that

$$
f(t) \leqslant b e^{\rho t}+C_{b}, \quad t \in \mathbf{R} .
$$

Now this leads naturally to the idea of comparing with some other function $g$ instead of the exponential function $g(t)=e^{t}$. So we might want to consider all numbers $a$ such that

$$
f(t) \leqslant g(a t)+C_{a}, \quad t \in \mathbf{R},
$$

and then take the infimum of all $a$. 
For reasons which will become clear when we come to the duality between order and type, it is desirable to change this inequality to

$$
f(t) \leqslant \frac{1}{a} g(a t)+C_{a}, \quad t \in \mathbf{R} .
$$

Now in the classical case, when $g(t)=e^{t}$, the factor $1 / a$ does not make any difference whatsoever, for in this case we see that for any $a>0$ and any $b>a$ there is a constant $C_{a, b}$ such that

$$
g(a t) \leqslant \frac{1}{b} g(b t)+C_{a, b} \quad \text { and } \quad \frac{1}{a} g(a t) \leqslant g(b t)+C_{a, b} .
$$

This implies that comparisons with $g(a t)$ and with $g(a t) / a$ give identical infima. But of course there exist functions $g$ such that this is not true (e.g., $g(t)=t$ ), and then (2.2) and (2.3) lead to different definitions of the order.

\section{Relative order and type of convex functions}

Definition 3.1. Let $f, g: E \rightarrow[-\infty,+\infty]$ be two functions defined on a real vector space $E$. We consider inequalities of the form

$$
f(x) \leqslant \frac{1}{a} g(a x)+c, \quad x \in E,
$$

where $a$ is a positive constant and $c$ a real constant. We shall call the infimum of all positive numbers $a$ such that (3.1) holds for some constant $c$ the order of $f$ relative to $g$, and denote it by $\rho=\operatorname{order}(f: g)$.

Examples. The motivating example is

$$
\operatorname{order}\left(t \mapsto e^{A t}: t \mapsto e^{t}\right)=A
$$

for all positive numbers $A$. Trivial examples are: $\operatorname{order}(a: b)=0$ if $a$ and $b$ are finite constants; $\operatorname{order}(f:+\infty)=0 ; \operatorname{order}(-\infty: g)=0$; $\operatorname{order}(f:-\infty)=+\infty$ except if $f$ is identically $-\infty$; order $(+\infty: g)=+\infty$ except if $g$ is identically $+\infty$.

If $g$ is convex, we know that

$$
\frac{1}{a} g(a x) \leqslant \frac{1-t}{a} g(0) \dot{+} \frac{t}{a} g(b x)=\left(\frac{1}{a}-\frac{1}{b}\right) g(0) \dot{+} \frac{1}{b} g(b x), \quad x \in E,
$$

if $0<a<b$ and $a x=(1-t) \cdot 0+t b x$, i. e., $t=a / b$. Here the sign $\dot{+}$ denotes upper addition, which is an extension of the usual addition from $\mathbf{R}^{2}$ to $[-\infty,+\infty]^{2}$; it satisfies $(+\infty) \dot{+}(-\infty)=+\infty$. Similarly we define lower addition as the extension of + which satisfies $(+\infty)+(-\infty)=-\infty$. If $g(0)=+\infty$ the inequality (3.2) is without interest, but if $g(0)<+\infty$, it shows that the inequality (3.1) for a particular $a$ implies the same inequality with $a$ replaced by $b$ for any $b>a$. The set of all numbers $a, 0<a<+\infty$, such that (3.1) holds is therefore an interval, either $[\rho,+\infty[$ or $] \rho,+\infty[$ for some $\rho \in[0,+\infty]$.

So although Definition 3.1 has a sense for all $f$ and $g$, it is often desirable to assume that $g$ is convex with $g(0)<+\infty$ : in this case the order determines the set of all $a$ for which (3.1) holds, with the exception of one point, the order itself. 
Lemma 3.2. Let $f_{y}$ denote the translate of $f$ by the vector $y: f_{y}(x)=f(x-y)$. If one of $f$ and $g$ is convex and real valued, then

$$
\operatorname{order}\left(f_{y}: g\right)=\operatorname{order}\left(f: g_{y}\right)=\operatorname{order}(f: g) \text {. }
$$

In particular order $\left(f_{y}: g_{y}\right)=\operatorname{order}(f: g)$ so that the order is translation invariant and can be defined on affine spaces as soon as one of the functions is convex and real valued.

Proof. If $f$ is convex and real valued, we know that

$$
f(x-y) \leqslant \frac{1}{b} f(b x)+\left(1-\frac{1}{b}\right) f(z)
$$

for any $b>1$, if we choose $z$ such that

$$
x-y=\frac{1}{b} b x+\left(1-\frac{1}{b}\right) z,
$$

i. e., if $z=-y /(1-1 / b)$. If $\operatorname{order}(f: g)=\rho$, there are numbers $a$ arbitrarily close to $\rho$ such that

$$
f(x) \leqslant \frac{1}{a} g(a x)+c .
$$

We then estimate $f$ as follows:

$$
f(x-y) \leqslant \frac{1}{b} f(b x)+\left(1-\frac{1}{b}\right) f(z) \leqslant \frac{1}{a b} g(a b x)+\frac{1}{b} c+\left(1-\frac{1}{b}\right) f(z) .
$$

Since $f(z)$ is finite and independent of $x$, this shows that $\operatorname{order}\left(f_{y}: g\right) \leqslant a b$, and since $b$ is arbitrarily close to 1 , we see that $\operatorname{order}\left(f_{y}: g\right) \leqslant \rho$. If we apply this result to $f_{y}$, translating by the vector $-y$, we get equality.

Similarly, if $g$ is convex and real valued, we can write

$$
f(x-y) \leqslant \frac{1}{a} g(a(x-y))+c \leqslant \frac{1}{a b} g(a b x)+\frac{1}{a}\left(1-\frac{1}{b}\right) g(z)+c,
$$

where $z=-a y /(1-1 / b)$, thus independent of $x$. This shows that, in this case also, $\operatorname{order}\left(f_{y}: g\right) \leqslant a b$ with $a b$ arbitrarily close to $\rho$.

It remains to consider $\operatorname{order}\left(f: g_{y}\right)$. The arguments are the same as for $\operatorname{order}\left(f_{y}: g\right)$; we omit the proof.

It is easy to give examples of functions with values in $]-\infty,+\infty]$ such that the order is not translation invariant:

Example. Let $f$ be the indicator function of the ball $r B$, i. e., let $f(x)=0$ when $|x| \leqslant r$ and $f(x)=+\infty$ otherwise. Similarly let $g$ be the indicator function of the ball $s B$. In the case where $0<s \leqslant r$ we get

$$
\frac{s}{r} \leqslant \operatorname{order}\left(f_{y}: g_{y}\right)=\frac{s+|y|}{r+|y|} \leqslant 1 .
$$

If $s>r>0$, we have

$$
\operatorname{order}\left(f_{y}: g_{y}\right)= \begin{cases}\frac{s-|y|}{r-|y|} \geqslant \frac{s}{r}>1 & \text { when }|y|<r \\ +\infty & \text { when }|y| \geqslant r .\end{cases}
$$

We now consider a generalisation of the notion of type in complex analysis. 
Definition 3.3. Given two functions $f, g: E \rightarrow[-\infty,+\infty]$ on a vector space $E$, we consider inequalities

$$
f(x) \leqslant b g(x)+c, \quad x \in E,
$$

where $b$ is a positive number. We define the type of $f$ relative to $g$ as the infimum of all positive numbers $b$ such that (3.3) holds for some constant $c$. We shall denote it by $\sigma=\operatorname{type}(f: g)$.

Example. The motivating example is

$$
\operatorname{type}\left(t \mapsto A e^{\rho t}: t \mapsto e^{\rho t}\right)=A \text {. }
$$

The two functions here are the growth functions of the entire functions $\exp \left(A z^{\rho}\right)$ and $\exp \left(z^{\rho}\right)$ if $\rho$ is a natural number, and then $A$ is the classical type with respect to order $\rho$.

If $g$ is bounded from below, the set of all numbers such that (3.3) holds is an interval, for as soon as $b_{1}>b$ we have

$$
b g(x)+c=b_{1} g(x)+c-\left(b_{1}-b\right) g(x) \leqslant b_{1} g(x)+c-\left(b_{1}-b\right) \inf g=b_{1} g(x)+c_{1} .
$$

Therefore, although the definition has a sense for all functions, it is clear that it will often be necessary to assume $g$ bounded from below. In this case the type determines all numbers $b$ for which (3.3) holds, except the number $\sigma$ itself.

Proximate orders are introduced to give functions of finite order normal type $(0<\sigma<+\infty)$; see Lelong \& Gruman [10, Appendix II]. The type with respect to a proximate order is a special case of Definition 3.3.

A generalisation of the classical order and type has been studied, e. g., by Sato $[\mathbf{1 1}]$ and Juneja, Kapoor \& Bajpai $[\mathbf{3}, 4]$. For given integers $p$ and $q$, they study the $(p, q)$-order defined as

$$
\rho_{p q}=\limsup \frac{\log ^{[p]} M(r)}{\log ^{[q]} r}=\limsup \frac{\log ^{[p-1]} f(t)}{\log ^{[q-1]} t},
$$

where $M(r)=\exp f(\log r)$. (Sato considered this only for $q=1$.) Here the brackets indicate iterations of the logarithm function. Now it is easy to see that the $(p, q)$ order is just order $\left(f_{q}: g_{p}\right)$ where $f_{q}(t)=f\left(\exp ^{[q-1]}(t)\right)$ and $g_{p}(t)=\exp ^{[p-1]}(t)$. Both $f_{q}$ and $g_{p}$ are convex. Their generalisation of the notion of type is, however, different from that of Definition 3.3. The $(p, q)$-type is

$$
T_{p q}=\limsup \frac{\log ^{[p-1]} M(r)}{\left(\log ^{[q-1]} r\right)^{\rho}}=\limsup \frac{\log ^{[p-2]} f(t)}{\left(\log ^{[q-2]} t\right)^{\rho}} .
$$

For $p \geqslant 3$ this is not the relative type of one convex function with respect to another, but rather an order: it is the order of $f\left(\exp ^{[q-2]} t^{1 / \rho}\right)$ with respect to $\exp ^{[p-2]}(t)$. Therefore our results on order generalise those of the authors mentioned, but our type is different, and some of the earlier results on type can be interpreted as orders in the framework of the present paper. 


\section{Order and type in duality}

The notion of order and type as defined in the last section are dual, or conjugate, to each other in the sense of convexity theory. We shall express duality here in terms of the Fenchel transformation: for any function $f: E \rightarrow[-\infty,+\infty]$ we define

$$
\widetilde{f}(\xi)=\sup _{x \in E}(\xi \cdot x-f(x)), \quad \xi \in E^{\prime} .
$$

Here $E$ is a real vector space, and $E^{\prime}$ is any fixed linear subspace of its algebraic dual $E^{*}$. The function $\widetilde{f}$ is called the Fenchel transform of $f$; other names are the Legendre transform of $f$, or the conjugate function. It is easy to see that $\widetilde{f}$ is convex, lower semicontinuous for the weak-star-topology $\sigma\left(E^{\prime}, E\right)$ and that it never takes the value $-\infty$ except when it is equal to $-\infty$ identically.

Points where $f(x)=+\infty$ do not influence the supremum in (4.1). We shall use this fact in the following way. Let $\operatorname{dom} f$ denote the set where $f(x)<+\infty$, the effective domain of $f$. Then for any set $M$ such that $\operatorname{dom} f \subset M \subset E$ we have

$$
\widetilde{f}(\xi)=\sup _{x \in M}(\xi \cdot x-f(x)), \quad \xi \in E^{\prime} .
$$

The inequality $\xi \cdot x \leqslant f(x) \dot{+} \widetilde{f}(\xi)$ is called Fenchel's inequality. Applying the transformation twice we get

$$
\widetilde{\widetilde{f}}(x)=\sup _{\xi \in E^{\prime}}(\xi \cdot x-\tilde{f}(\xi)) \leqslant f(x), \quad x \in E .
$$

Thus always $\widetilde{\widetilde{f}} \leqslant f$; the equality $\widetilde{\widetilde{f}}=f$ holds if and only if $f$ is convex, lower semicontinuous for the weak topology $\sigma\left(E, E^{\prime}\right)$, and takes the value $-\infty$ only if it is $-\infty$ identically. More generally, it follows that $\widetilde{\widetilde{f}}$ is the maximal convex lower semicontinuous minorant of $f$ which never takes the value $-\infty$ except when it is the constant $-\infty$. For these properties of the Fenchel transform see Rockafellar [11]. Of course $\widetilde{\widetilde{f}}$ depends on the choice of $E^{\prime}$; if $E^{\prime}=\{0\}$, then $\widetilde{\widetilde{f}}$ is the constant inf $f$. If $E=\mathbf{R}^{n}$ it is natural to take $E^{\prime}=E^{*} \cong \mathbf{R}^{n}$; if $E$ is a topological vector space one usually takes $E^{\prime}$ as the topological dual of $E$.

Proposition 4.1. Let $f, g: E \rightarrow[-\infty,+\infty]$ be two functions on a vector space $E$. Then

$$
\operatorname{type}(\widetilde{g}: \widetilde{f}) \leqslant \operatorname{order}(f: g) \text {. }
$$

Proof. If $\operatorname{order}(f: g)<A$, then $f(x) \leqslant g(a x) / a+c$ for some number $a<A$, and we deduce that $\widetilde{f}(\xi) \geqslant \widetilde{g}(\xi) / a-c$, which we write as $\widetilde{g}(\xi) \leqslant a \widetilde{f}(\xi)+a c$. Therefore $\operatorname{type}(\widetilde{g}: \widetilde{f}) \leqslant a<A$.

Proposition 4.2. If $f, g: E \rightarrow[-\infty,+\infty]$ are two functions on a vector space $E$, then

$$
\operatorname{order}(\widetilde{g}: \widetilde{f}) \leqslant \operatorname{type}(f: g) \text {. }
$$

Proof. If type $(f: g)<A$ there are numbers $a<A$ and $c$ such that $f(x) \leqslant a g(x)+c$. We take the transformation to obtain $\widetilde{f}(\xi) \geqslant a \widetilde{g}(\xi / a)-c$, which can be written as $\widetilde{g}(\xi) \leqslant \widetilde{f}(a \xi) / a+c / a$. Therefore $\operatorname{order}(\widetilde{g}: \widetilde{f}) \leqslant a<A$. 
Theorem 4.3. Let $f, g: E \rightarrow[-\infty,+\infty]$ be two functions on a vector space $E$ such that $\widetilde{\widetilde{f}}=f$ and $\widetilde{\widetilde{g}}=g$. Then

$$
\operatorname{order}(\widetilde{g}: \widetilde{f})=\operatorname{type}(f: g) \quad \text { and } \quad \operatorname{type}(\widetilde{g}: \widetilde{f})=\operatorname{order}(f: g) \text {. }
$$

Proof. We just combine Propositions 4.1 and 4.2 .

Corollary 4.4. Let $E=\mathbf{R}^{n}$ and choose $E^{\prime}=\mathbf{R}^{n}$. Let $f, g: \mathbf{R}^{n} \rightarrow[-\infty,+\infty]$ be two functions satisfying the hypotheses of the theorem. Assume in addition that $f$ is finite in a neighbourhood of the origin and grows faster than any linear function, and that $g$ is not the constant $+\infty$. Then

$$
\operatorname{order}(f: g)=\limsup _{\xi \rightarrow \infty} \frac{\widetilde{g}(\xi)}{\widetilde{f}(\xi)} .
$$

Proof. If $f \leqslant M$ for $|x|<\varepsilon$ we obtain $\tilde{f}(\xi) \geqslant \varepsilon|\xi|-M$. Therefore $0<\tilde{f}<+\infty$ in a neighbourhood of $\infty$, and $\lim \tilde{f}=+\infty$, so that the type is given by

$$
\operatorname{type}(\widetilde{g}: \widetilde{f})=\limsup _{\xi \rightarrow \infty} \frac{\widetilde{g}(\xi)}{\widetilde{f}(\xi)} .
$$

\section{The infimal convolution}

The infimal convolution is an important operation in convexity theory. It is actually dual to addition, so many problems can be reduced to simple questions using the Fenchel transformation, but it is often preferable to work directly with it. In this section we just recall the definition. Later, in sections 10 and 11, we shall return to it to make a more detailed study.

The infimal convolution of two functions $f, g: E \rightarrow[-\infty,+\infty]$ is defined by

$$
f \square g(x)=\inf _{y}(f(y) \dot{+} g(x-y)), \quad x \in E .
$$

Here the sign $\dot{+}$ denotes upper addition; see section 3. The Fenchel transform of an infimal convolution is

$$
(f \square g)^{\sim}(\xi)=\widetilde{f}(\xi)+\widetilde{g}(\xi), \quad \xi \in E^{\prime},
$$

where + is lower addition. (It might seem strange that we get lower addition here, for in general $f \dot{+} g$ is convex when both $f$ and $g$ are convex, but not $f+g$. However, in this case $\tilde{f}+\widetilde{g}$ equals $\widetilde{f} \dot{+} \widetilde{g}$ except when it is constant, so it is always convex.)

\section{The order of an entire function}

Let $F \in \mathcal{O}\left(\mathbf{C}^{n}\right)$ be an entire function. We shall measure its growth by

$$
f(t)=\sup _{z}\left[\log |F(z)| ; z \in \mathbf{C}^{n},|z| \leqslant e^{t}\right], \quad t \in \mathbf{R} .
$$


Here $|z|$ can be any norm on $\mathbf{C}^{n}$, or even an arbitrary function which is complex homogeneous of degree one and positive on the unit sphere. We shall refer to $f$ as the growth function of $F$. In view of Hadamard's three-circle-theorem, $f$ is convex and increasing, and we shall write

$$
\operatorname{order}(F: G)=\operatorname{order}(f: g)
$$

by abuse of language if $F, G$ are two entire functions and $f, g$ are their growth functions.

One may ask which convex increasing functions can appear as growth functions. A necessary condition is that of Hayman [2]: for a transcendental entire function $F \in \mathcal{O}(\mathbf{C})$, we have

$$
\limsup _{t \rightarrow+\infty} f^{\prime \prime}(t) \geqslant H
$$

where $H$ is an absolute constant satisfying $0.18<H \leqslant 0.25$. Kjellberg [9] proved that $0.24<H<0.25$. (A similar statement holds for polynomials.) Another necessary condition is as follows. Define

$$
f_{1}(t)=\sup _{j \in \mathbf{N}}(j t-\widetilde{f}(j)), \quad t \in \mathbf{R} .
$$

(The epigraph of $f_{1}$ is the smallest polygon which contains the epigraph of $f$ and whose sides have integer slopes.) Then there is a constant $C$ such that

$$
f(t)-C \leqslant f_{1}(t) \leqslant f(t), \quad t \in \mathbf{R} .
$$

Moreover the best constant $C$ satisfies $\log 2 \leqslant C \leqslant \log 3$ (Kiselman [7, Proposition 5.1]). These two results are not unrelated, for the latter implies that $H \geqslant(8 C)^{-1}$. With $C=\log 3$ this gives $H \geqslant(8 \log 3)^{-1} \approx 0.11$, which is much weaker than the Hayman-Kjellberg result. On the other hand, that statement does not say anything about tangents of integer slope.

If two entire functions $F$ and $G$ are given, we consider their expansions in terms of homogeneous polynomials $P_{j}$ and $Q_{j}$ :

$$
F(z)=\sum_{0}^{\infty} P_{j}(z), \quad G(z)=\sum_{0}^{\infty} Q_{j}(z),
$$

and ask whether we can determine $\operatorname{order}(F: G)$ from knowledge of the growth of $\left|P_{j}\right|$ and $\left|Q_{j}\right|$. It turns out that this is so. For the classical order, when $G=\exp$, this is well known. We shall see in section 12 that this is not necessarily true for type $(F: G)$.

So let $F$ be given with an expansion in terms of homogeneous polynomials $P_{j}$. Cauchy's inequalites say that

$$
\left|P_{j}(z)\right| \leqslant \exp (f(\log |z|)),
$$

but the homogeneity of $P_{j}$ also gives

$$
\left|P_{j}(z)\right|=\frac{|z|^{j}}{e^{j t}} P_{j}\left(e^{t} z /|z|\right) \leqslant \frac{|z|^{j}}{e^{j t}} \exp (f(t))=|z|^{j} \exp (f(t)-j t)
$$


for all real $t$ and all $z \in \mathbf{C}^{n}$. We take the infimum over all $t$ and get

$$
\left|P_{j}(z)\right| \leqslant|z|^{j} e^{-\tilde{f}(j)}
$$

We define the norm $\left\|P_{j}\right\|$ of the homogeneous polynomial $P_{j}$ as

$$
\left\|P_{j}\right\|=\sup _{|z| \leqslant 1}\left|P_{j}(z)\right|, \quad j \in \mathbf{N} .
$$

(When $n=1$ we have a Taylor expansion $F(z)=\sum a_{j} z^{j}$ and $\left\|P_{j}\right\|=\left|a_{j}\right|$.) We next define a function $p: \mathbf{R} \rightarrow]-\infty,+\infty]$ as

$$
p(j)= \begin{cases}-\log \left\|P_{j}\right\| & \text { when } j \in \mathbf{N} ; \\ +\infty & \text { when } j \in \mathbf{R} \backslash \mathbf{N} .\end{cases}
$$

We shall call $p$ the coefficient function of $F$. Cauchy's inequalities become just $\left\|P_{j}\right\| \leqslant \exp (-\widetilde{f}(j))$, or more concisely

$$
p \geqslant \tilde{f} \quad \text { on } \mathbf{R} \text {. }
$$

This implies of course that $\widetilde{p} \leqslant \widetilde{\widetilde{f}}=f$. Note also that

$$
\exp \widetilde{p}(\log r)=\sup _{j \in \mathbf{N}} \sup _{|z| \leqslant r}\left|P_{j}(z)\right|
$$

We now ask for inequalities in the other direction. To describe this result we need an auxiliary function $K$ which is defined as follows:

$$
K(t)= \begin{cases}-\log \left(1-e^{t}\right), & t<0 \\ +\infty, & t \geqslant 0 .\end{cases}
$$

We have $K(t) \geqslant-\log (-t)$ when $t<0$ (a good approximation for small $|t|$ ) and $K(t) \geqslant e^{t}$ for all $t$ (a good approximation for $t \ll 0$ ). The Fenchel transform of $K$ is

$$
\widetilde{K}(\tau)= \begin{cases}\tau \log \tau-(\tau+1) \log (\tau+1), & \tau>0 \\ 0, & \tau=0 \\ +\infty, & \tau<0\end{cases}
$$

We note that

$$
-1-\log (\tau+1) \leqslant \widetilde{K}(\tau) \leqslant-\log (\tau+1), \quad \tau \geqslant 0 .
$$

The inverse of $K$ is given by $K^{-1}(s)=-K(-s)$ for $s>0$ : this means that the graph of $K$ is symmetric around the line $s+t=0$. This symmetry corresponds to the functional equation $\widetilde{K}(1 / \tau)=\widetilde{K}(\tau) / \tau, \tau>0$, for the transform. 
Theorem 6.1. Let $F$ be an entire function in $\mathbf{C}^{n}$ and define $f$ and $p$ by (6.1) and (6.2), respectively. Then

$$
\widetilde{p} \leqslant f \leqslant \widetilde{p} \square K \quad \text { on } \mathbf{R} \text {. }
$$

Proof. We have just noted that Cauchy's inequalities give $\widetilde{p} \leqslant \widetilde{\widetilde{f}}=f$. To estimate $f$ from above we write

$$
|F(z)| \leqslant \sum\left\|P_{j}\right\| \cdot|z|^{j} \leqslant \sum \exp (-p(j)+j t),
$$

where $t=\log |z|$. We shall apply Fenchel's inequality $j t \leqslant p(j) \dot{+} \widetilde{p}(t)$ in the form

$$
-p(j)+j t \leqslant j s+\widetilde{p}(t-s) .
$$

This gives

$$
f(t) \leqslant \log \sum e^{-p(j)+j t} \leqslant \log \sum e^{j s+\tilde{p}(t-s)} .
$$

We observe that

$$
\sum_{j \in \mathbf{N}} e^{j s}=\frac{1}{1-e^{s}}=e^{K(s)}
$$

if $s<0$, which is why we introduced $K$. Thus $f(t) \leqslant \widetilde{p}(t-s)+K(s)$ for all $t \in \mathbf{R}$ and all $s<0$. Now for $s \geqslant 0, K(s)=+\infty$, so then the inequality also holds, and we can let $s$ vary over the whole real axis:

$$
f(t) \leqslant \inf _{s}(\widetilde{p}(t-s) \dot{+} K(s))=(\widetilde{p} \square K)(t), \quad t \in \mathbf{R} .
$$

This proves the theorem.

The inequalities (6.6) say that the graph of $f$ is in a strip whose lower boundary is the polygon defined by $\widetilde{p}$ and whose upper boundary is given by $\widetilde{p} \square K$. Since $K(-\log 2)=\log 2$, the width of this strip is at most $\sqrt{2} \log 2 \approx 0.98<1$.

Applying the Fenchel transformation to all members of $(6.6)$ we get:

$$
p \geqslant \widetilde{\widetilde{p}} \geqslant \widetilde{f} \geqslant \widetilde{\widetilde{p}}+\widetilde{K},
$$

where $\widetilde{K}$ can be estimated by $(6.5)$.

For lacunary series we can state:

Theorem 6.2. Let $F$ be lacunary: $P_{j}=0$ for $j \notin J$. Then

$$
\widetilde{p} \leqslant f \leqslant \widetilde{p} \square K_{J} \quad \text { on } \mathbf{R},
$$

where

$$
K_{J}(s)=\log \left(\sum_{j \in J} e^{j s}\right) .
$$

Proof. Just restrict summation in the proof of Theorem 6.1 to $j \in J$.

It could be noted here that for any convex function $H$ which is positive on the negative half-axis and tends to $+\infty$ as $t<0, t \rightarrow 0$, there exists an infinite set $J \subset \mathbf{N}$ such that $K_{J} \leqslant H$.

Theorem 6.1 implies that the norms of the homogeneous polynomials $P_{j}$ can serve just as well as the growth function $f$ to determine the order of $F$ relative to any other function. More precisely we have: 
Corollary 6.3. Let $F$ be an entire function on $\mathbf{C}^{n}$, let $f$ be its growth function defined by (6.1), and let $p$ be its coefficient function defined by (6.2). Assume that $F$ is not a polynomial. Then

$$
\operatorname{order}(f: \widetilde{p})=\operatorname{order}(\widetilde{p}: f)=1
$$

Proof. From (6.6) we get immediately

$$
\operatorname{order}(\widetilde{p}: f) \leqslant 1, \quad \operatorname{order}(f: \widetilde{p} \square K) \leqslant 1 .
$$

Now $\widetilde{p} \square K(t) \leqslant \widetilde{p}(t+1)+K(-1)$ and Lemma 3.2 shows that the translation of $\tilde{p}$ does not influence the order, neither does of course the additive constant $K(-1)$. Therefore

$$
\operatorname{order}(f: \widetilde{p}) \leqslant \operatorname{order}(f: \widetilde{p} \square K) \leqslant 1 .
$$

It follows from Corollary 4.4 that $\operatorname{order}(f: f)=1$. By submultiplicativity,

$$
1=\operatorname{order}(f: f) \leqslant \operatorname{order}(f: \widetilde{p}) \cdot \operatorname{order}(\widetilde{p}: f) \leqslant 1
$$

so that all orders must be one. (When $F$ is a polynomial, $\operatorname{order}(f: f)=0$ and $\operatorname{order}(f: \widetilde{p})=\operatorname{order}(\widetilde{p}: f)=0$. $)$

Corollary 6.4. Let $F$ be an entire function in $\mathbf{C}^{n}$, with expansion

$$
F(z)=\sum P_{j}(z)
$$

in terms of homogeneous polynomials $P_{j}$. Let $f$ be its growth function defined by (6.1) and let $p$ be its coefficient function defined by (6.2). Let $g: \mathbf{R} \rightarrow[-\infty,+\infty]$ be any function which satisfies $\widetilde{\widetilde{g}}=g$. Then

$$
\operatorname{order}(f: g)=\operatorname{order}(\widetilde{p}: g)=\operatorname{type}(\widetilde{g}: \widetilde{\widetilde{p}}) \text {. }
$$

When $F$ is not a polynomial and $g$ not identically $+\infty$, the order is also given by

$$
\limsup _{j \rightarrow+\infty} \frac{\widetilde{g}(j)}{p(j)}
$$

Proof. Using Corollary 6.3 we can write

$$
\operatorname{order}(f: g) \leqslant \operatorname{order}(f: \widetilde{p}) \cdot \operatorname{order}(\widetilde{p}: g)=\operatorname{order}(\widetilde{p}: g)
$$

provided $F$ is not a polynomial. Similarly

$$
\operatorname{order}(\widetilde{p}: g) \leqslant \operatorname{order}(\widetilde{p}: f) \cdot \operatorname{order}(f: g)=\operatorname{order}(f: g) \text {. }
$$

The last equality in (6.8) follows from Theorem 4.3. If $F$ is a polynomial, one can verify (6.8) directly, using $\widetilde{p} \leqslant f \leqslant \widetilde{p}+\log N$, where $N$ is the number of terms in the expansion (see Theorem 6.2). The only possibilities are then $\operatorname{order}(f: g)=0,+\infty$. 
We finally have, if $F$ is not a polynomial,

$$
\operatorname{type}(\widetilde{g}: \widetilde{\widetilde{p}})=\limsup \frac{\widetilde{g}(\tau)}{\widetilde{\widetilde{p}}(\tau)}=\limsup \frac{\widetilde{g}(j)}{p(j)} .
$$

The last equality holds because on the one hand $\widetilde{\widetilde{p}} \leqslant p$, on the other hand $\widetilde{\widetilde{p}}=p$ in a sequence of integers tending to infinity, and $\widetilde{\widetilde{p}}$ is affine in between these points.

Formula (6.9) generalises the classical formula for the order

$$
\rho=\limsup \frac{j \log j}{-\log \left|a_{j}\right|}
$$

of an entire function $\sum a_{j} z^{j}$. Indeed, when the comparison function is $g(t)=e^{t}$, then $\widetilde{g}(j)=j \log j-j$.

The $(p, q)$-order of Juneja, Kapoor \& Bajpai [3, Theorem 1] is determined in terms of the coefficients by the formula

$$
\rho_{p, q}=\limsup \frac{\log ^{[p-1]} j}{\log ^{[q-1]}\left(-(1 / j) \log \left|a_{j}\right|\right)} ;
$$

we state it only for $p>q \geqslant 1$ here. Sato [11] proved this for $q=1$. In the latter case (6.9) is a generalisation. For $q \geqslant 2$, however, this is not so, since then $f\left(\exp ^{[q-1]} t\right)$ is used as the growth function and consequently defines another relation between the coefficients $a_{j}($ or $p(j))$ and $f$.

It could also be noted here that Corollary 6.4 generalises the classical result that the order can be calculated from the dominant term in a series expansion $\sum a_{j} z^{j}$. Indeed, with $t=\log |z|$ the maximal term is just

$$
\sup _{j}\left|a_{j} z^{j}\right|=\exp \sup _{j}(j t-p(j))=\exp \widetilde{p}(t) .
$$

Another classical result does not generalise easily, however. The classical order is also given by

$$
\rho=\limsup \frac{\log N(r)}{\log r}
$$

where $N(r)$ is the rank of the maximal term. In our notation this would be one of the numbers $j=j_{t}$ such that the equality $j t=p(j)+\widetilde{p}(t)$ holds. We can take it as a one-sided derivative $j_{t}=\widetilde{p}^{\prime}(t)$ (the undeterminacy is unimportant). When $\widetilde{p}$ is like exp, its derivative is also like exp, so that

$$
\widetilde{p}(t)=\left(\widetilde{p} \circ\left(\widetilde{p}^{\prime}\right)^{-1}\right)\left(j_{t}\right) \approx j_{t},
$$

yielding

$$
\rho=\limsup \frac{\log j_{t}}{t}
$$

but in general $\widetilde{p} \circ\left(\widetilde{p}^{\prime}\right)^{-1}$ is far from the identity. More precisely, $\operatorname{order}(\widetilde{p}: g) \leqslant$ $\operatorname{order}\left(\widetilde{p}^{\prime}: g\right)$ as soon as the function $g$ satisfies the following condition: for any $a>1$ there is a constant $c$ such that

$$
t g(t) \leqslant \frac{1}{a} g(a t)+c
$$

This follows easily from the inequality $\widetilde{p}(t) \leqslant \widetilde{p}(0)+t \widetilde{p}^{\prime}(t)$. But the other inequality, $\operatorname{order}(\widetilde{p}: g) \geqslant \operatorname{order}\left(\widetilde{p}^{\prime}: g\right)$, holds only under special conditions on $\widetilde{p}$.

When we have two entire functions we can state: 
Corollary 6.5. Let $F, G$ be two entire functions in $\mathbf{C}^{n}$, with expansions

$$
F(z)=\sum P_{j}(z), \quad G(z)=\sum Q_{j}(z)
$$

in terms of homogeneous polynomials $P_{j}, Q_{j}$. Let $p$ and $q$ denote their coefficient functions defined by (6.2). Then

$$
\operatorname{order}(F: G)=\operatorname{order}(\widetilde{p}: \widetilde{q})=\operatorname{type}(\widetilde{\widetilde{q}}: \widetilde{\widetilde{p}}) .
$$

Proof. The proof is analogous to that of Corollary 6.4.

We can also define a growth function related to the growth of an entire function on polydisks, and to Taylor expansions in terms of monomials. Let us define

$$
f(x)=\sup _{\left|z_{j}\right| \leqslant \exp x_{j}} \log |F(z)|, \quad x \in \mathbf{R}^{n}
$$

if $F$ is an entire function on $\mathbf{C}^{n}$. Then $f$ is convex in $\mathbf{R}^{n}$. The function $F$ has an expansion

$$
F(z)=\sum_{k \in \mathbf{N}^{n}} A_{k} z^{k}, \quad z \in \mathbf{C}^{n}
$$

where $z^{k}$ denotes the monomial $z_{1}^{k_{1}} \cdots z_{n}^{k_{n}}$ of multidegree $k=\left(k_{1}, \ldots, k_{n}\right)$ and total degree $k_{1}+\cdots+k_{n}$. Cauchy's inequalities now say that, for $r=\left(r_{1}, \ldots, r_{n}\right)$ with $r_{j}>0$,

$$
\left|A_{k}\right| r^{k} \leqslant \sup _{\left|z_{j}\right| \leqslant r_{j}}|F(z)|=e^{f(x)}, \quad x_{j}=\log r_{j} .
$$

This gives $\left|A_{k}\right| \leqslant \exp (f(x)-k \cdot x)$ for all $x \in \mathbf{R}^{n}$, and therefore, after variation of $x$,

$$
\left|A_{k}\right| \leqslant \exp (-\widetilde{f}(k)), \quad k \in \mathbf{N}^{n} .
$$

We introduce in analogy with (6.2)

$$
a(k)= \begin{cases}-\log \left|A_{k}\right| & \text { when } k \in \mathbf{N}^{n} \\ +\infty & \text { when } k \in \mathbf{R}^{n} \backslash \mathbf{N}^{n} .\end{cases}
$$

Then $a \geqslant \tilde{f}$ and $\widetilde{a} \leqslant \widetilde{\widetilde{f}}=f$. Next define $K_{n}(x)=K\left(x_{1}\right)+\cdots+K\left(x_{n}\right)$ for $x \in \mathbf{R}^{n}$. In complete analogy with Theorem 6.1 we have:

Theorem 6.6. Let $F$ be an entire function in $\mathbf{C}^{n}$ and define the growth function $f$ and the coefficient function a by (6.10) and (6.11), respectively. Then

$$
\widetilde{a} \leqslant f \leqslant \widetilde{a} \square K_{n} \quad \text { on } \mathbf{R}^{n} .
$$

A variant of the growth function can be defined as follows. Let $u$ be a plurisubharmonic function on $\mathbf{C}^{n}$ which is extremal in the set $a<u(z)<b$ : it is the regularised supremum of all plurisubharmonic functions $\varphi$ in a neighbourhood of the closure of 
$\{z ; a<u(z)<b\}$ which satisfy $\varphi(z) \leqslant a$ when $u(z) \leqslant a$ and $\varphi(z) \leqslant b$ when $u(z) \leqslant b$. We suppose that $\{z ; u(z)<b\}$ is bounded, and define for $F \in \mathcal{O}\left(\mathbf{C}^{n}\right)$

$$
f_{u}(t)=\sup _{z}(\log |F(z)| ; u(z)<t) .
$$

Then $f_{u}$ is easily seen to be convex on $\left.] a, b\right]$. (The growth function $f$ defined by (6.1) is with respect to the extremal plurisubharmonic function $u(z)=\log |z|$ provided $|z|$ is a norm or more generally $\log |z|$ is plurisubharmonic; if not, we can replace it by a suitable plurisubharmonic minorant.)

We can for instance ask whether a holomorphic function on a complex analytic variety $X$ admits an entire extension of the same order: if $F \in \mathcal{O}(X), X \subset \mathbf{C}^{n}$, does there exist an entire function $G \in \mathcal{O}\left(\mathbf{C}^{n}\right)$ such that $\operatorname{order}(G: F)=1$ ? Here it might be natural to define the growth functions $f_{u}$ and $g_{v}$ of $F$ and $G$ with respect to extremal functions $u$ on $X$ and $v$ on $\mathbf{C}^{n}$, respectively.

\section{A geometric characterisation of the relative order}

In this section we shall give a geometric interpretation of the relative order. Let $E$ be a real vector space. We consider two hyperplanes $E \times\{0\}$ and $E \times\{1\}$ in the Cartesian product $E \times \mathbf{R}$. Now let two functions $\left.\left.f_{0}, f_{1}: E \rightarrow\right]-\infty,+\infty\right]$ be given. We consider them as defined on $E \times\{0\}$ and $E \times\{1\}$ respectively, and want to find a function $F: E \times \mathbf{R} \rightarrow]-\infty,+\infty]$ extending them, i.e., a function such that

$$
F(x, j)=f_{j}(x), \quad x \in E, \quad j=0,1 .
$$

If the $f_{j}$ are convex, a solution is of course the supremum of all convex minorants to the function $f(x, t)=f_{t}(x)$ if $t=0$ or $t=1, f(x, t)=+\infty$ otherwise. This solution is the largest possible: it majorises all others. But it is of no interest outside the slab $\{0 \leqslant t \leqslant 1\}$, since it is always $+\infty$ there.

In general there is no unique solution, for we can always add $t^{2}-t$ to any given solution. We can however write down an explicit formula for an extremal solution.

Proposition 7.1. Let $E$ be a real vector space and $E^{\prime}$ a subspace of its algebraic dual. Let $\left.\left.f_{0}, f_{1}: E \rightarrow\right]-\infty,+\infty\right]$ be two given convex functions which are lower semicontinuous with respect to $\sigma\left(E, E^{\prime}\right)$. We assume that they are not identically plus infinity. Then the extrapolation problem

$$
\left\{\begin{array}{l}
\text { Find } F: E \times \mathbf{R} \rightarrow]-\infty,+\infty] \\
F(x, j)=f_{j}(x), \quad \text { such that }
\end{array}\right.
$$

has a solution

$$
\begin{aligned}
F(x, t) & =\sup _{\xi}\left[\xi \cdot x-(1-t) \widetilde{f}_{0}(\xi)-t \widetilde{f}_{1}(\xi) ; \xi \in \operatorname{dom} \widetilde{f}_{0} \cup \operatorname{dom} \tilde{f}_{1}\right] \\
& =\sup _{\xi}\left[\xi \cdot x-\left((1-t) \tilde{f}_{0}(\xi) \dot{+} t \widetilde{f}_{1}(\xi)\right) ; \xi \in E^{\prime}\right], \quad(x, t) \in E \times \mathbf{R} .
\end{aligned}
$$

This solution is extremal in the sense that any convex solution $G$ which is lower semicontinuous in $x$ satisfies $G \leqslant F$ in $\{0 \leqslant t \leqslant 1\}$ and $G \geqslant F$ outside this slab. 
Proof. First a word about the definition of $F$. We note that the function $t \mapsto t \cdot(+\infty)$ is convex on the whole real line, if we define $0 \cdot(+\infty)=0$. We also note that in the first expression defining $F$ at most one of the three terms is infinite, for we have $-\infty<\widetilde{f}_{j} \leqslant+\infty$ everywhere, and at most one of them is allowed to be plus infinity in the set of $\xi$ which we use. Therefore $F$ is well defined, and it is convex as a supremum of functions of $(x, t)$ each of which is an affine function plus possibly one function of the form $(t-1) \cdot(+\infty)$ or $(-t) \cdot(+\infty)$. Moreover, for $t=j$ the function $F$ assumes the values $\widetilde{\widetilde{f}}_{j}(x)=f_{j}(x), j=0,1$, in view of (4.2). Therefore it is a convex solution to the extension problem. It is of course not lower semicontinuous in all variables, but it is lower semicontinuous in $x$ for fixed $t$.

Now let $G$ be another convex solution to the problem. Let us consider

$$
\widetilde{G}_{t}(\xi)=\sup _{x \in E}(\xi \cdot x-G(x, t)), \quad t \in \mathbf{R}, \quad \xi \in E^{\prime}
$$

It is concave in $t$ for fixed $\xi$, for it is the marginal function of a concave function of $(x, t)$. It satisfies moreover $\widetilde{G}_{j}(\xi)=\widetilde{f}_{j}(\xi), j=0,1$. If we assume that $G$ is lower semicontinuous in $x$ and $>-\infty$, we also have

$$
G(x, t)=\sup _{\xi}\left(\xi \cdot x-\widetilde{G}_{t}(\xi)\right) .
$$

When $0<t<1$ we have

$$
\operatorname{dom}\left((1-t) \tilde{f}_{0}+t \tilde{f}_{1}\right)=\operatorname{dom} \tilde{f}_{0} \cap \operatorname{dom} \widetilde{f}_{1} \subset \operatorname{dom} \widetilde{f}_{0} \cup \operatorname{dom} \widetilde{f}_{1}
$$

The fact that $\widetilde{G}_{j}=\widetilde{f}_{j}$ for $j=0,1$ implies that $\widetilde{G}_{t} \geqslant(1-t) \widetilde{f}_{0}+t \widetilde{f}_{1}$. This gives $G \leqslant F$.

When $t<0$ or $t>1$ the concavity in $t$ gives $\widetilde{G}_{t} \leqslant(1-t) \widetilde{f}_{0}+t \widetilde{f}_{1}$ and then $G \geqslant F$. This establishes the extremal character of the solution $F$.

We now ask how far outside the slab $\{0 \leqslant t \leqslant 1\}$ we can obtain a real-valued solution to the extrapolation problem. An answer is given by the next theorem.

Theorem 7.2. Let $\left.\left.f_{0}, f_{1}: E \rightarrow\right]-\infty,+\infty\right]$ be two given convex and lower semicontinuous functions. Assume that $f_{0}(0)<+\infty$. If the extrapolation problem (7.1) admits a convex solution $F$ which is finite at a point $(0, t)$ with $t$ satisfying $1<t<+\infty$, then

$$
\operatorname{order}\left(f_{1}: f_{0}\right) \leqslant \frac{t}{t-1} \text {. }
$$

Conversely, if $1 \leqslant \operatorname{order}\left(f_{1}, f_{0}\right)=\rho<+\infty$, then the extrapolation problem has a lower semicontinuous convex solution $F$ with $F(0, t)<+\infty$ for all $t$ with $0 \leqslant t<$ $\rho /(\rho-1)$. Thus if we denote by $b$ the supremum of all numbers $t$ such that there exists a solution $F$ which is finite at the point $(0, t)$, then

$$
\operatorname{order}\left(f_{1}: f_{0}\right)=\rho=\frac{b}{b-1}=b^{\prime}
$$

(We assume $1 \leqslant \rho<+\infty$ and $1<b \leqslant+\infty$.) 
Proof. If $F$ is convex we have

$$
F(x, 1) \leqslant \frac{1}{a} F(a x, 0) \dot{+}\left(1-\frac{1}{a}\right) F(0, t),
$$

where $a>1$ is chosen so that

$$
(x, 1)=\frac{1}{a}(a x, 0)+\left(1-\frac{1}{a}\right)(0, t) \in E \times \mathbf{R},
$$

i. e., $a=t /(t-1)$. Now if $F(0, t)<+\infty$ this inequality shows that

$$
f_{1}(x) \leqslant \frac{1}{a} f_{0}(a x)+c
$$

in other words that $\operatorname{order}\left(f_{1}: f_{0}\right) \leqslant a=t /(t-1)$.

Conversely, if $\operatorname{order}\left(f_{1}, f_{0}\right) \leqslant \rho$ with $1 \leqslant \rho<+\infty$, then the solution $F$ defined by (7.2) has the desired properties. We need only estimate $F$ as follows. For any $a>\rho$ we know that $f_{1}(x) \leqslant f_{0}(a x) / a+c$ which gives $\widetilde{f}_{1} \geqslant a^{-1} \widetilde{f}_{0}-c$. In particular we see that $\operatorname{dom} \widetilde{f}_{0} \supset \operatorname{dom} \widetilde{f}_{1}$. For any $t<a /(a-1)$ we can write, letting $\xi$ vary in $\operatorname{dom} \widetilde{f}_{0}$,

$$
\begin{aligned}
F(x, t) & \leqslant \sup _{\xi}\left[\xi \cdot x-(1-t) \widetilde{f}_{0}(\xi)-t\left(a^{-1} \widetilde{f}_{0}(\xi)-c\right)\right]= \\
& =\sup _{\xi}\left[\xi \cdot x-(1-t+t / a) \widetilde{f}_{0}(\xi)\right]+t c= \\
& =(1-t+t / a) \sup _{\xi}\left[(1-t+t / a)^{-1} \xi \cdot x-\widetilde{f}_{0}(\xi)\right]+t c=\delta f_{0}(x / \delta)+t c,
\end{aligned}
$$

where $\delta$ is the positive number $1-t+t / a$. Now, since we assume that $f_{0}(0)<+\infty$, this shows that $F(0, t)$ is finite for all $t \in[0, a /(a-1)[$, and since $a$ is any number larger than $\rho$, the function is finite for all $t \in[0, b[$.

For real-valued functions the geometry is particularly simple:

Corollary 7.3. Let $\left.\left.f_{0}, f_{1}: E \rightarrow\right]-\infty,+\infty\right]$ be two functions as in Proposition 7.1 and assume in addition that one of them is real valued. If the extrapolation problem (7.1) admits a convex solution $F$ which is finite at some point $(x, t)$ with $t$ satisfying $1<t<+\infty$, then

$$
\operatorname{order}\left(f_{1}: f_{0}\right) \leqslant \frac{t}{t-1} \text {. }
$$

Conversely, if $1 \leqslant \operatorname{order}\left(f_{1}, f_{0}\right)=\rho<+\infty$, then the extrapolation problem has a lower semicontinuous convex solution $F$ which is real valued in the slab

$$
E \times] 0, \rho^{\prime}\left[=\left\{(x, t) \in E \times \mathbf{R} ; 0<t<\rho^{\prime}\right\},\right.
$$

where $\rho^{\prime}=\rho /(\rho-1) ; 1<\rho^{\prime} \leqslant+\infty$.

Therefore the relative order of $f_{1}$ with respect to $f_{0}$ is determined by, and determines, the maximal slab $E \times] 0, b[$ in which our extrapolation problem has a solution. 
Proof. Suppose $f_{j}$ is real valued $(j=0$ or $j=1)$. It is clear that if a solution $F$ is finite at some point $(x, s)$ with $s>1$, then $F$ is finite in the convex hull of the union of $(x, s)$, some point $(y, 0)$ where $f_{0}$ is finite, and the hyperplane $E \times\{j\}$. This convex hull contains the slab $E \times] 0, s[$. Thus Theorem 7.2 implies Corollary 7.3.

It follows again (cf. Lemma 3.2) that the notion of relative order is translation invariant for real-valued convex functions (at least when $1 \leqslant \rho+\infty$ ). Indeed, the slabs are invariant under transformations $(x, t) \mapsto(x-(1-t) y-t z, t)$ for all $y$ and $z$; these transformations correspond to translations $f_{0} \mapsto f_{0, y}$ and $f_{1} \mapsto f_{1, z}$.

\section{An extension problem for holomorphic functions}

In this section we shall first characterise the classical order in terms of an extension property of holomorphic functions. Then we pass to the relative order.

Theorem 8.1. An entire function $F \in \mathcal{O}\left(\mathbf{C}^{n}\right)$ is of order at most $\rho(1 \leqslant \rho<+\infty)$ if and only if there exists a holomorphic function $H$ in the cylinder

$$
\Omega=\left\{(z, w) \in \mathbf{C}^{n} \times \mathbf{C} ;|w|<e^{\rho^{\prime}}\right\}
$$

where $\rho^{\prime}=\rho /(\rho-1)$, satisfying

$$
|H(z, w)| \leqslant e^{|z|} \quad \text { for } z \in \mathbf{C}^{n},|w| \leqslant 1,
$$

and

$$
H(z, e)=F(z) \quad \text { for } z \in \mathbf{C}^{n} \text {. }
$$

Proof. Suppose such an $H$ exists. Then putting

$$
h(s, t)=\sup \left[\log |H(z, w)| ;|z| \leqslant e^{s},|w| \leqslant e^{t}\right], \quad s \in \mathbf{R}, t<\rho^{\prime},
$$

we get a convex function of $(s, t)$ which satisfies $h(s, 0) \leqslant e^{s}$ and $h(s, 1) \geqslant f(s)$. Therefore, applying Corollary 7.3 with $f_{0}(s)=h(s, 0)$ and $f_{1}(s)=h(s, 1)$, we can write

$$
\operatorname{order}(F: \exp ) \leqslant \operatorname{order}\left(f_{1}: \exp \right) \leqslant \operatorname{order}\left(f_{1}: f_{0}\right) \cdot \operatorname{order}\left(f_{0}: \exp \right) \leqslant \rho
$$

In the other direction the results of section 7 give only convex, not holomorphic, solutions to the extrapolation problem. But it turns out that there is an explicit solution in terms of power series.

We expand $F$ in a series of homogeneous polynomials:

$$
F(z)=\sum P_{j}(z)
$$

Then we just define

$$
H(z, w)=\sum P_{j}(z)(w / e)^{m_{j}}
$$


where the integers $m_{j}$ are chosen large enough to make (8.1) true. This means that we take

$$
\left\|P_{j}\right\| e^{-m_{j}} \leqslant \frac{1}{j !}
$$

On the other hand, we do not want to take them unnecessarily large, so we prescribe that

$$
\log \left\|P_{j}\right\|+\log j ! \leqslant m_{j}<\log \left\|P_{j}\right\|+\log j !+1
$$

unless $P_{j}=0$ in which case the choice of $m_{j}$ is immaterial, so we may take $m_{j}=0$.

Since $F$ is of order $\rho$, we know that for any $a>\rho$ there is an estimate $f(t) \leqslant$ $e^{a t}+C_{a}$, which implies that

$$
\widetilde{f}(\tau) \geqslant \frac{\tau}{a}\left(\log \frac{\tau}{a}-1\right)-C_{a}
$$

and

$$
-\log \left\|P_{j}\right\|=p(j) \geqslant \tilde{f}(j) \geqslant \frac{j}{a}\left(\log \frac{j}{a}-1\right)-C_{a}
$$

This estimate shows that the series defining $H$ converges uniformly on any compact subset of $\Omega$. In fact, the series defining $H$ converges uniformly for $|z| \leqslant R_{1}<R$ and $|w| \leqslant r_{1}<r$ if $\left\|P_{j}\right\| R^{j}(r / e)^{m_{j}} \rightarrow 0$. Substituting the expression for $m_{j}$ we see that this is true if $(\log r-1) \log j !-p(j) \log r+j \log R \rightarrow-\infty$. Now this holds for all positive $R$ if

$$
\frac{(\log r-1) \log j !-p(j) \log r}{j} \rightarrow-\infty
$$

Using finally the estimate (8.4) for $p$ and the inequality $j ! \leqslant j^{j}$ for the factorial function we see that this follows if

$$
(\log r-1) \log j-\frac{1}{a}\left(\log \frac{j}{a}-1\right) \log r \rightarrow-\infty,
$$

which in turn is true if $\log r<a /(a-1)$. Here the only condition is that $a>\rho$, so the series defining $H$ converges locally uniformly in the set $\log |w|<\rho /(\rho-1)$.

Theorem 8.2. Let two transcendental entire functions $F, G \in \mathcal{O}\left(\mathbf{C}^{n}\right)$ be given, and let $1 \leqslant \rho<+\infty$. We define an open set $\Omega$ in the space of $n+1$ variables as

$$
\Omega=\left\{(z, w) \in \mathbf{C}^{n} \times \mathbf{C} ;|w|<e^{\rho^{\prime}}\right\}
$$

where $\rho^{\prime}=\rho /(\rho-1)\left(1<\rho^{\prime} \leqslant+\infty\right)$. For a holomorphic function $H$ in $\Omega$ we denote by $h_{w}$ the growth function of the partial function $z \mapsto H(z, w)$. Let $K$ denote the function defined by (6.4). Then the following five conditions are equivalent.

(a) $\operatorname{order}(G: F) \leqslant \rho$.

(b) There exists a holomorphic function $H \in \mathcal{O}(\Omega)$ satisfying

$$
h_{w} \leqslant f \square K \quad \text { when }|w|=1, \quad \text { and } \quad g \leqslant h_{w} \square K \quad \text { when }|w|=e .
$$


$\left(b^{\prime}\right)$ There exists a holomorphic function $H \in \mathcal{O}(\Omega)$ satisfying $H(z, 1)=F(z)$,

$$
f \leqslant h_{w} \square K, \quad \text { and } \quad h_{w} \leqslant f \square K \quad \text { when }|w|=1,
$$

and

$$
g \leqslant h_{w} \square K \quad \text { when }|w|=e .
$$

(c) There exists a holomorphic function $H \in \mathcal{O}(\Omega)$ satisfying $H(z, e)=G(z)$ and

$$
h_{w} \leqslant f \square K \quad \text { when }|w|=1 .
$$

$\left(c^{\prime}\right)$ There exists a holomorphic function $H \in \mathcal{O}(\Omega)$ satisfying $H(z, e)=G(z)$,

$$
g \leqslant h_{w} \square K \quad \text { and } \quad h_{w} \leqslant g \square K \quad \text { when }|w|=e,
$$

and

$$
h_{w} \leqslant f \square K \quad \text { when }|w|=1 .
$$

In particular, $\operatorname{order}(H(\cdot, w): F) \leqslant 1$ for $|w|=1$ and $\operatorname{order}(G: H(\cdot, w)) \leqslant 1$ for $|w|=e$ if $H$ is the holomorphic function whose existence is guaranteed by (b), ( $\left.\mathrm{b}^{\prime}\right)$ or $\left(\mathrm{c}^{\prime}\right)$.

Proof. The proof that (b) implies (a) and that (c) implies (a) is just like the easy direction in the proof of Theorem 8.1. If $H$ is a holomorphic function satisfying (b) or (c) we let $h$ be the growth function of two real variables defined by (8.3); it is related to the $h_{w}$ by the formula $h(s, t)=\sup _{|w|=e^{t}} h_{w}(s)$. By submultiplicativity we then have

$$
\operatorname{order}(g: f) \leqslant \operatorname{order}(g: h(\cdot, 1)) \cdot \operatorname{order}(h(\cdot, 1): h(\cdot, 0)) \cdot \operatorname{order}(h(\cdot, 0): f) \leqslant \rho .
$$

It is also clear that $\left(\mathrm{b}^{\prime}\right)$ implies $(\mathrm{b})$ and that $\left(\mathrm{c}^{\prime}\right)$ implies $(\mathrm{c})$.

For the proof of (a) implies (b') we expand $F$ and $G$ in terms of homogeneous polynomials:

$$
F(z)=\sum_{j \in \mathbf{N}} P_{j}(z), \quad G(z)=\sum_{j \in \mathbf{N}} Q_{j}(z),
$$

and define

$$
H(z, w)=\sum_{j \in \mathbf{N}} P_{j}(z) w^{m_{j}}+(w-1) \sum_{j \notin J} P_{j}^{*}(z) w^{m_{j}},
$$

where $J$ is the set of all $j \in \mathbf{N}$ such that $p(j) \leqslant \widetilde{\widetilde{p}}(j)+\log 3, p$ being the coefficient function of $F$ defined by (6.2). Moreover $m_{j}$ are suitable integers and $P_{j}^{*}$ homogeneous polynomials of degree $j$ and norm $\left\|P_{j}^{*}\right\|=\frac{1}{3} \exp (-\widetilde{\widetilde{p}}(j)) \geqslant\left\|P_{j}\right\|$. Let $p_{w}$ denote the coefficient function of the entire function $H(\cdot, w)$. Consider first $|w|=1$ : when $j \in J$ we have $p_{w}(j)=p(j)$, and when $j \notin J$ we can estimate as follows:

$$
\left\|P_{j} w^{m_{j}}+(w-1) P_{j}^{*} w^{m_{j}}\right\| \leqslant\left\|P_{j}\right\|+2\left\|P_{j}^{*}\right\| \leqslant 3\left\|P_{j}^{*}\right\|=\exp (-\widetilde{\widetilde{p}}(j)),
$$


so that $p_{w}(j) \geqslant \widetilde{\widetilde{p}}(j)$ when $j \notin J$. Therefore we have $p_{w} \geqslant \widetilde{\widetilde{p}}$ everywhere and $p_{w}=p$ in $J$, which implies $\widetilde{p}_{w}=\widetilde{p}$ for $|w|=1$. Thus in view of Theorem 6.1,

$$
h_{w} \leqslant \widetilde{p}_{w} \square K=\widetilde{p} \square K \leqslant f \square K \quad \text { as well as } \quad f \leqslant \widetilde{p} \square K=\widetilde{p}_{w} \square K \leqslant h_{w} \square K .
$$

This far the numbers $m_{j}$ play no role; we shall now choose them to get the right kind of growth of $H(\cdot, w)$ for $|w|=e$. When $|w|=e$ and $j \in J$ we have $p_{w}(j)=$ $p(j)-m_{j} \leqslant \widetilde{\widetilde{p}}(j)-m_{j}+\log 3$. The homogeneous part of degree $j \notin J$ in $H(z, w)$ can be estimated as

$$
\begin{aligned}
\left\|P_{j} w^{m_{j}}+(w-1) P_{j}^{*} w^{m_{j}}\right\| \geqslant e^{m_{j}}\left(\left\|(w-1) P_{j}^{*}\right\|-\left\|P_{j}\right\|\right) & \\
& \geqslant e^{m_{j}}(e-2)\left\|P_{j}^{*}\right\|=\frac{e-2}{3} \exp \left(m_{j}-\widetilde{\widetilde{p}}(j)\right),
\end{aligned}
$$

which gives

$$
\begin{array}{ll}
p_{w}(j)=p(j)-m_{j} \leqslant \widetilde{\widetilde{p}}(j)-m_{j}+\log 3 \leqslant \widetilde{\widetilde{p}}(j)-m_{j}+2, & j \in J, \\
p_{w}(j) \leqslant \widetilde{\widetilde{p}}(j)-m_{j}-\log \left(\frac{e-2}{3}\right) \leqslant \widetilde{\widetilde{p}}(j)-m_{j}+2, & j \notin J .
\end{array}
$$

We shall now choose the integers $m_{j}$ as follows. If $\widetilde{\widetilde{p}}(j)=+\infty$ (this can happen for finitely many numbers $j$ only), then also $\widetilde{\widetilde{q}}(j)=+\infty$ and we choose $m_{j}=0$. If $\widetilde{\widetilde{p}}(j)<$ $+\infty$ we choose $m_{j}$ as the smallest non-negative integer which is $\geqslant \widetilde{\widetilde{p}}(j)-\widetilde{\widetilde{q}}(j)+2$. Thus in all cases $p_{w} \leqslant \widetilde{q}$ for every $w$ with $|w|=e$, so that $\widetilde{q} \leqslant \widetilde{p}_{w}$ and we get

$$
g \leqslant \widetilde{q} \square K \leqslant \widetilde{p}_{w} \square K \leqslant h_{w} \square K, \quad|w|=e .
$$

Finally we have to make sure that $H$ is holomorphic in all of $\Omega$. To prove this it is enough to prove that

$$
\left\|P_{j}\right\| R^{j} r^{m_{j}} \rightarrow 0 \quad \text { and } \quad\left\|P_{j}^{*}\right\| R^{j} r^{m_{j}} \rightarrow 0
$$

as $j \rightarrow \infty$ for all $R$ and all $r<e^{\rho^{\prime}}$. This in turn follows if we can prove that

$$
\frac{m_{j} \log r-p(j)}{j} \rightarrow-\infty \quad \text { and } \quad \frac{m_{j} \log r-p^{*}(j)}{j} \rightarrow-\infty
$$

We shall use the fact that type $(\widetilde{\widetilde{p}}: \widetilde{\widetilde{q}})=\operatorname{order}(g: f) \leqslant \rho$, which yields an inequality $\widetilde{\widetilde{p}} \leqslant a \widetilde{q}+C_{a}$ for every $a>\rho$. If $m_{j}=0$, the first expression in (8.5) is at most $-\widetilde{\widetilde{p}}(j) / j$ which certainly tends to $-\infty$. If $m_{j}>0$, it can be estimated by (it suffices to consider $r>1$ )

$$
\frac{m_{j} \log r-p(j)}{j} \leqslant \frac{(\widetilde{\widetilde{p}}(j)-\widetilde{\widetilde{q}}(j)+3) \log r-\widetilde{\widetilde{p}}(j)}{j} \leqslant \frac{\widetilde{\widetilde{q}}(j)(a \log r-\log r-a)+O(1)}{j}
$$

which tends to $-\infty$ if $\log r<a /(a-1)$. 
If $m_{j}=0$, the second expression in $(8.5)$ is $-p^{*}(j) / j=-(\widetilde{\widetilde{p}}(j)-\log 3) / j$ which tends to $-\infty$; if $m_{j}>0$, it can be estimated by

$$
\begin{aligned}
\frac{m_{j} \log r-p^{*}(j)}{j} & \leqslant \frac{(\widetilde{\widetilde{p}}(j)-\widetilde{\widetilde{q}}(j)+3) \log r-\widetilde{\widetilde{p}}(j)+\log 3}{j} \\
& \leqslant \frac{\widetilde{\widetilde{q}}(j)(a \log r-\log r-a)+O(1)}{j},
\end{aligned}
$$

which tends to $-\infty$ as soon as $\log r<a /(a-1)$; here again $a$ is any number greater than $\rho$. This proves that the series defining $H$ converges locally uniformly in $\Omega$ and finishes the proof of $\left(b^{\prime}\right)$.

The proof that (a) implies $\left(c^{\prime}\right)$ is similar to that of Theorem 8.1. This time we define

$$
H(z, w)=\sum_{j \in \mathbf{N}} Q_{j}(z)(w / e)^{n_{j}}
$$

where we shall choose integers $n_{j}$. Then obviously $H(z, e)=G(z)$. For $|w|=e$ we have $p_{w}(j)=q(j)$. This gives $\widetilde{p}_{w}=\widetilde{q}$ and therefore, for all $w$ with $|w|=e$,

$$
h_{w} \leqslant \widetilde{p}_{w} \square K=\widetilde{q} \square K \leqslant g \square K \quad \text { as well as } \quad g \leqslant \widetilde{q} \square K=\widetilde{p}_{w} \square K \leqslant h_{w} \square K \text {. }
$$

For $|w|=1$, on the other hand, we obtain

$$
\left\|Q_{j}(w / e)^{n_{j}}\right\|=\exp \left(-n_{j}-q(j)\right) \leqslant \exp \left(-n_{j}-\widetilde{\widetilde{q}}(j)\right) .
$$

Thus, when $|w|=1$ we have

$$
p_{w}(j)=q(j)+n_{j} \geqslant \widetilde{\widetilde{q}}(j)+n_{j} .
$$

We now choose $n_{j}$ so that $p_{w} \geqslant \widetilde{\widetilde{p}}$, which implies $\widetilde{p}_{w} \leqslant \widetilde{p}$ and yields the estimate

$$
h_{w} \leqslant \widetilde{p}_{w} \square K \leqslant \widetilde{p} \square K \leqslant f \square K .
$$

To be explicit, if $\widetilde{\widetilde{p}}(j)=+\infty$, then $\widetilde{\widetilde{q}}(j)=+\infty$ and we take $n_{j}=0$; if $\widetilde{\widetilde{p}}(j)<+\infty$, we take $n_{j}$ as the smallest non-negative integer greater than or equal to $\widetilde{\widetilde{p}}(j)-\widetilde{\widetilde{q}}(j)$. This guarantees that $p_{w} \geqslant \widetilde{\widetilde{p}}$ and gives the estimate above. On the other hand, $n_{j}$ is not too large, which will ensure that $\left\|Q_{j}\right\| R^{j}(r / e)^{n_{j}}$ tends to zero for every $R$ and every $r<e^{\rho^{\prime}}$ and hence that $H$ is holomorphic in $\Omega$. The calculation is very similar to the one we just carried out in the case of $\left(b^{\prime}\right)$ and is omitted.

\section{Whittaker's decomposition theorem}

In analogy with (2.1) one defines classically the lower order of an entire function as

$$
\lambda=\liminf _{r \rightarrow+\infty} \sup _{|z|=r} \frac{\log \log |h(z)|}{\log r} .
$$

The obvious generalisation is: 
Definition 9.1. Whenever the order of $g$ relative to $f$ is defined, we define the lower relative order of $f$ with respect to $g$ as

$$
\text { lower } \operatorname{order}(f: g)=1 / \operatorname{order}(g: f) \in[0,+\infty] \text {. }
$$

According to the terminology of Whittaker [13] functions with $\lambda=\rho<+\infty$ are said to be of regular growth. He shows that if an entire function has gaps in the sense that

$$
F(z)=\sum a_{k} z^{m_{k}}, \quad z \in \mathbf{C},
$$

with $\lim \sup \left(\log m_{k+1} / \log m_{k}\right)>1$, then $F$ is not of regular growth. And conversely, if a function is of irregular growth, then it can be represented, for every $\mu$ satisfying $\lambda<\mu<\rho$, as a sum $F=F_{0}+F_{1}$ where $F_{0}$ is of order at most $\mu$ and $F_{1}$ has gaps as indicated.

We shall prove here a complete analogue of Whittaker's results for the relative order. The results will also generalise Theorems 4 and 5 of Juneja, Kapoor \& Bajpai [3]. Since there is no such thing as absolutely large gaps in a world of relative growth, the size of the gaps will have to be measured by that same function $g$ which we use to define order and lower order. If an entire function has gaps which are large as measured by $g$, then it is of irregular growth with respect to $g$, meaning that $\operatorname{order}(f: g) \cdot \operatorname{order}(g: f)>1$, and conversely, if a function is of irregular growth with respect to $g$, then it can be decomposed as in the classical case. The gaps will be measured as follows.

Definition 9.2. Let $\varphi$ be a function defined on R. Assume that $\varphi>0$. Then the $\varphi$-gauge of an interval $[\sigma, \tau]$ is

$$
J_{\varphi}(\sigma, \tau)=\sup _{0 \leqslant s \leqslant 1} \frac{(1-s) \varphi(\sigma)+s \varphi(\tau)}{\varphi((1-s) \sigma+s \tau)} .
$$

Example. If $\varphi(\tau)=\tau \log \tau-\tau$, then $J_{\varphi}(\sigma, \tau) \approx \log \tau / \log \sigma$ for $\sigma \leqslant \tau$, in the precise sense that $J_{\varphi}(\sigma, \tau) \log \sigma / \log \tau$ tends to 1 as $\sigma \rightarrow+\infty$ uniformly for all $\tau \geqslant \sigma$. Since this $\varphi$ is the Fenchel transform of the classical comparison function exp, we see that the results below will give Whittaker's when $g=\exp$.

Theorem 9.3. Let $g: \mathbf{R} \rightarrow \mathbf{R}$ be an increasing convex function which grows faster than any linear function, and let $F$ be an entire function with an expansion $F=$ $\sum P_{m_{k}}$ in terms of homogeneous polynomials of degree $m_{k}$. We assume that $\operatorname{order}(f$ : $g)<+\infty$. Then

$$
\operatorname{order}(f: g) \cdot \operatorname{order}(g: f) \geqslant \limsup _{k \rightarrow+\infty} J_{\tilde{g}}\left(m_{k}, m_{k+1}\right) .
$$

In particular, if $F$ has gaps which are large as measured by $\widetilde{g}$, then $F$ is of irregular growth relative to $g$.

Proof. Denote the limit superior in the statement by $\alpha(1 \leqslant \alpha \leqslant+\infty)$. Given any number $\beta<\alpha$ we can choose $s_{k}$ such that

$$
\frac{\left(1-s_{k}\right) \widetilde{g}\left(m_{k}\right)+s_{k} \widetilde{g}\left(m_{k+1}\right)}{\widetilde{g}\left(\left(1-s_{k}\right) m_{k}+s_{k} m_{k+1}\right)} \geqslant \beta
$$


for an infinite sequence of indices $k$. Let $\rho=\operatorname{order}(f: g)=\operatorname{order}(\widetilde{p}: g)=\operatorname{type}(\widetilde{g}: \widetilde{\widetilde{p}})$. Then we know that $\widetilde{g} \leqslant(\rho+\varepsilon) \widetilde{\widetilde{p}}$ for large arguments, so we can write

$$
\beta \leqslant(\rho+\varepsilon) \frac{\left(1-s_{k}\right) \widetilde{\widetilde{p}}\left(m_{k}\right)+s_{k} \widetilde{\widetilde{p}}\left(m_{k+1}\right)}{\widetilde{g}\left(\left(1-s_{k}\right) m_{k}+s_{k} m_{k+1}\right)}=(\rho+\varepsilon) \frac{\widetilde{\widetilde{p}}\left(j_{k}\right)}{\widetilde{g}\left(j_{k}\right)},
$$

where we have defined $j_{k}=\left(1-s_{k}\right) m_{k}+s_{k} m_{k+1}$, and where the last equality holds because $\widetilde{\widetilde{p}}$ is affine in the interval $\left[m_{k}, m_{k+1}\right]$; this follows from the fact that there are gaps, which means that $p$ is $+\infty$ on the interior of that interval. Hence

$$
\operatorname{order}(g: f)=\operatorname{order}(g: \widetilde{p})=\operatorname{type}(\widetilde{\widetilde{p}}: \widetilde{g}) \geqslant \frac{\beta}{\rho+\varepsilon}
$$

If we now let $\beta$ tend to $\alpha$ we get $\operatorname{order}(g: f) \cdot \operatorname{order}(f: g) \geqslant \alpha$.

Theorem 9.4. Let $F \in \mathcal{O}\left(\mathbf{C}^{n}\right)$ be an entire function and let $g$ be any convex increasing real-valued function on $\mathbf{R}$. Assume that $F$ is of finite order and of irregular growth relative to $g$, i. e.,

$$
+\infty>\rho=\operatorname{order}(f: g)>\lambda=1 / \operatorname{order}(g: f) \geqslant 0 .
$$

Then for every $\mu$ satisfying $\lambda<\mu<\rho$ there is a decomposition $F=F_{0}+F_{1}$ where $\operatorname{order}\left(F_{0}: g\right) \leqslant \mu$ and where $F_{1}$ has gaps which are of $\widetilde{g}$-gauge at least $\mu / \lambda$ asymptotically.

Proof. We define

$$
J=\{j \in \mathbf{N} ; p(j) \geqslant \widetilde{g}(j) / \mu\}, \quad F_{0}=\sum_{j \in J} P_{j} .
$$

Let $p_{0}$ denote the function which agrees with $p$ on $J$ and is $+\infty$ elsewhere. Then $p_{0} \geqslant \widetilde{g} / \mu$ on $J$ and therefore everywhere, which implies $\widetilde{\widetilde{p}}_{0} \geqslant \widetilde{g} / \mu$, and we see that $F_{0}$ has order at most $\mu$ :

$$
\operatorname{order}\left(F_{0}: g\right)=\operatorname{order}\left(\widetilde{p}_{0}: g\right)=\operatorname{type}\left(\widetilde{g}: \widetilde{p}_{0}\right) \leqslant \mu \text {. }
$$

We shall prove that $F_{1}=F-F_{0}$ has gaps as indicated. Let $p_{1}$ denote the function which agrees with $p$ on $\mathbf{N} \backslash J$ and is $+\infty$ elsewhere. Then $\widetilde{\widetilde{p}}_{1}$ is affine in every interval which does not meet $\mathbf{N} \backslash J$. By the definition of lower order, there exists a sequence $\left(j_{k}\right)$ such that

$$
\widetilde{\widetilde{p}}\left(j_{k}\right) \geqslant \frac{1}{\lambda+1 / k} \widetilde{g}\left(j_{k}\right) .
$$

Let $m_{k}$ be the largest element in $\mathbf{N} \backslash J$ which is smaller than $j_{k}$, and let $n_{k}$ be the smallest element in $\mathbf{N} \backslash J$ which is larger than $j_{k}$. Since $m_{k}, n_{k}$ are elements of $\mathbf{N} \backslash J$, we know that $\widetilde{\widetilde{p}}_{1}\left(m_{k}\right) \leqslant p_{1}\left(m_{k}\right)=p\left(m_{k}\right)<\widetilde{g}\left(m_{k}\right) / \mu$ and similarly at $n_{k}$. Since the interval $] m_{k}, n_{k}$ [ does not meet $\mathbf{N} \backslash J$, the function $\widetilde{\widetilde{p}}_{1}$ is affine on $\left[m_{k}, n_{k}\right]$. Then if 
we define $s$ so that $(1-s) m_{k}+s n_{k}=j_{k}$ we have $(1-s) \widetilde{\widetilde{p}}_{1}\left(m_{k}\right)+s \widetilde{\widetilde{p}}_{1}\left(n_{k}\right)=\widetilde{\widetilde{p}}_{1}\left(j_{k}\right)$, and

$$
\begin{aligned}
J_{\tilde{g}}\left(m_{k}, n_{k}\right) \geqslant \frac{(1-s) \widetilde{g}\left(m_{k}\right)+s \widetilde{g}\left(n_{k}\right)}{\widetilde{g}\left(j_{k}\right)} \geqslant & \frac{(1-s) \mu \widetilde{\widetilde{p}}_{1}\left(m_{k}\right)+s \mu \widetilde{\widetilde{p}}_{1}\left(n_{k}\right)}{(\lambda+1 / k) \widetilde{\widetilde{p}}_{1}\left(j_{k}\right)} \\
& =\frac{\mu \widetilde{\widetilde{p}}_{1}\left(j_{k}\right)}{(\lambda+1 / k) \widetilde{\tilde{p}}_{1}\left(j_{k}\right)}=\frac{\mu}{\lambda+1 / k} \rightarrow \frac{\mu}{\lambda}
\end{aligned}
$$

Thus the gaps as measured by $\widetilde{g}$ are at least $\mu / \lambda$ asymptotically; we have proved our claim.

We shall now discuss an analogous result for Taylor expansions $\sum A_{k} z^{k}$. We have to modify the measure of gaps. Let $i_{A}$ denote the indicator function of a set $A$; it is zero in $A$ and $+\infty$ in the complement.

Definition 9.5. Let $\varphi$ be a function defined on $\mathbf{R}^{n}$. For $M \subset \mathbf{N}^{n}$ we define

$$
\varphi_{M}=\left(\varphi+i_{\mathbf{N}^{n} \backslash M}\right) \approx
$$

the convex minorant of the function which agrees with $\varphi$ in $\mathbf{N}^{n} \backslash M$ and is $+\infty$ in $M$ as well as in $\mathbf{R}^{n} \backslash \mathbf{N}^{n}$. Then the $\varphi$-gauge of $M$ is

$$
J_{\varphi, M}(\xi)=\varphi_{M}(\xi) / \varphi(\xi), \quad \xi \in \mathbf{R}^{n}, \quad 0<\varphi(\xi)<+\infty .
$$

Note that we define $J_{\varphi, M}$ only at points where $0<\varphi(\xi)<+\infty$.

Theorem 9.6. Let $g: \mathbf{R}^{n} \rightarrow \mathbf{R}$ be a convex function, and let $F \in \mathcal{O}\left(\mathbf{C}^{n}\right)$ be an entire function with Taylor expansion $F(z)=\sum A_{k} z^{k}$. We assume that $0<\widetilde{g} \leqslant+\infty$ and that $F$ is of finite order relative to $g$. If $F$ has gaps which are large as measured by $\widetilde{g}$, then $F$ is of irregular growth relative to $g$. Quantitatively we express this as follows. Let $M$ denote the set of all $k \in \mathbf{N}^{n}$ such that $A_{k}=0$. Then

$$
\operatorname{order}(f: g) \cdot \operatorname{order}(g: f) \geqslant \limsup _{\xi \rightarrow \infty} J_{\tilde{g}, M}(\xi)
$$

where $\xi \rightarrow \infty$ means that $\widetilde{g}(\xi) \rightarrow+\infty$.

Proof. We write $\widetilde{g}_{M}=(\widetilde{g})_{M}$ for the function defined by (9.3) with $\varphi=\widetilde{g}$. Denote by $\alpha$ the limit superior in the statement of the theorem $(1 \leqslant \alpha \leqslant+\infty)$ and fix a number $\beta<\alpha$. Then there is a sequence $\left(\xi_{j}\right)$ such that $0<\widetilde{g}\left(\xi_{j}\right)<+\infty, \widetilde{g}\left(\xi_{j}\right) \rightarrow+\infty$ and such that

$$
\widetilde{g}_{M}\left(\xi_{j}\right) / \widetilde{g}\left(\xi_{j}\right) \geqslant \beta \text {. }
$$

We know that

$$
\rho=\operatorname{order}(f: g)=\operatorname{order}(\widetilde{a}: g)=\operatorname{type}(\widetilde{g}: \widetilde{\widetilde{a}}),
$$

so that for any positive $\varepsilon$ there is a constant $c$ such that $\widetilde{g} \leqslant(\rho+\varepsilon) \widetilde{\widetilde{a}}+c$. Now $\widetilde{\widetilde{a}} \leqslant a$, so $\widetilde{g} \leqslant(\rho+\varepsilon) a+c$, which implies that

$$
\widetilde{g}+i_{\mathbf{N}^{n} \backslash M} \leqslant(\rho+\varepsilon) a+c,
$$


for $a=+\infty$ in $M \cup\left(\mathbf{R}^{n} \backslash \mathbf{N}^{n}\right)$. This gives, by the definition of $\widetilde{g}_{M}$,

$$
\widetilde{g}_{M} \leqslant(\rho+\varepsilon) \widetilde{\widetilde{a}}+c
$$

so

$$
\beta \leqslant \frac{\widetilde{g}_{M}\left(\xi_{j}\right)}{\widetilde{g}\left(\xi_{j}\right)} \leqslant \frac{(\rho+\varepsilon) \widetilde{\widetilde{a}}\left(\xi_{j}\right)+c}{\widetilde{g}\left(\xi_{j}\right)} .
$$

Now $\widetilde{g}\left(\xi_{j}\right) \rightarrow+\infty$ so the constant $c$ does not interfere. We get finally

$$
\operatorname{order}(g: f)=\operatorname{type}(\widetilde{\widetilde{a}}: \widetilde{g}) \geqslant \frac{\beta}{\rho+\varepsilon}
$$

Since $\beta /(\rho+\varepsilon)$ is arbitrarily close to $\alpha / \rho$, this finishes the proof.

Theorem 9.7. Let $F \in \mathcal{O}\left(\mathbf{C}^{n}\right)$ be an entire function with growth function $f$ defined by (6.10), and let $g$ be any convex real-valued function on $\mathbf{R}^{n}$ with $\widetilde{g}>0$. Assume that $F$ is of finite order and of irregular growth relative to $g$, i. e.,

$$
+\infty>\rho=\operatorname{order}(f: g)>\lambda=1 / \operatorname{order}(g: f) \geqslant 0 .
$$

Then for every $\mu$ satisfying $\lambda<\mu<\rho$ there is a decomposition $F=F_{0}+F_{1}$ where $\operatorname{order}\left(F_{0}: g\right) \leqslant \mu$ and where $F_{1}$ has gaps which are of $\widetilde{g}$-gauge at least $\mu / \lambda$ asymptotically in the sense that $\lim \sup J_{\tilde{g}, K}(\xi) \geqslant \mu / \lambda$, where the limit superior is taken to mean that $\widetilde{g}(\xi) \rightarrow+\infty$ if $\lambda>0$, and to mean that $a(\xi) \rightarrow+\infty$ when $\lambda=0$.

Proof. Define

$$
\begin{gathered}
K=\left\{k \in \mathbf{N}^{n} ; a(k) \geqslant \widetilde{g}(k) / \mu\right\} \\
F_{0}(z)=\sum_{k \in K} A_{k} z^{k}, \quad \text { and } \quad F_{1}(z)=\sum_{k \in \mathbf{N}^{n} \backslash K} A_{k} z^{k} .
\end{gathered}
$$

Let $a_{0}$ be the function which agrees with $a$ in $K$ and is $+\infty$ outside, and let $a_{1}$ be the function which agrees with $a$ in $\mathbf{N}^{n} \backslash K$ and is $+\infty$ outside. Then $a \geqslant \tilde{g} / \mu$ in $K$ so $a_{0} \geqslant \widetilde{g} / \mu$ in $K$ and therefore in all of $\mathbf{R}^{n}$. This implies that $\widetilde{\widetilde{a}}_{0} \geqslant \widetilde{g} / \mu$ and hence

$$
\operatorname{order}\left(F_{0}: g\right)=\operatorname{order}\left(\widetilde{a}_{0}: g\right)=\operatorname{type}\left(\widetilde{g}: \widetilde{\widetilde{a}}_{0}\right) \leqslant \mu \text {. }
$$

In $\mathbf{N}^{n} \backslash K$ we have the inequality $\mu a<\widetilde{g}$; therefore also $\mu a_{1}<\widetilde{g}$ there, for $a_{1}=a$ in $\mathbf{N}^{n} \backslash K$. This implies $\mu a_{1} \leqslant \widetilde{g}+i_{\mathbf{N}^{n} \backslash K}$. By definition of the function $\widetilde{g}_{K}=(\widetilde{g})_{K}$ we therefore deduce

$$
\mu \widetilde{\widetilde{a}}_{1} \leqslant\left(\widetilde{g}+i_{\mathbf{N}^{n} \backslash K}\right)^{\approx}=\widetilde{g}_{K}
$$

So finally, for all $\xi$ such that $0<\widetilde{g}(\xi)<+\infty$,

$$
J_{\tilde{g}, K}(\xi)=\frac{\widetilde{g}_{K}(\xi)}{\widetilde{g}(\xi)} \geqslant \mu \frac{\widetilde{\widetilde{a}}_{1}(\xi)}{\widetilde{g}(\xi)} \geqslant \mu \frac{\widetilde{\widetilde{a}}(\xi)}{\widetilde{g}(\xi)}
$$


It remains to be proved that $\widetilde{\widetilde{a}}_{1} / \widetilde{g} \geqslant 1 / \lambda$ in a suitable sequence $\left(\xi_{j}\right)$. In view of the definition of the lower order there exists a sequence $\left(\xi_{j}\right)$ such that

$$
\widetilde{\widetilde{a}}\left(\xi_{j}\right)>\frac{1}{\lambda+1 / j} \widetilde{g}\left(\xi_{j}\right)+j
$$

in particular $\widetilde{\widetilde{a}}\left(\xi_{j}\right)>j$. Indeed, type $(\widetilde{\widetilde{a}}: \widetilde{g})=\operatorname{order}(g: \widetilde{a})=\operatorname{order}(g: f)=1 / \lambda$. So the expression $\widetilde{\widetilde{a}}_{1}\left(\xi_{j}\right) / \widetilde{g}\left(\xi_{j}\right)$ is at least

$$
\frac{\widetilde{\widetilde{a}}\left(\xi_{j}\right)}{\widetilde{g}\left(\xi_{j}\right)} \geqslant \frac{1}{\lambda+1 / j} \frac{\widetilde{g}\left(\xi_{j}\right)}{\widetilde{g}\left(\xi_{j}\right)}=\frac{1}{\lambda+1 / j} .
$$

Thus $\lim \sup J_{\tilde{g}, K}\left(\xi_{j}\right) \geqslant \mu / \lambda$ as claimed; we have $a\left(\xi_{j}\right) \geqslant \widetilde{\widetilde{a}}\left(\xi_{j}\right) \rightarrow+\infty$. If $\lambda>0$ we also have $\widetilde{g}\left(\xi_{j}\right) \rightarrow+\infty$, for type $(\widetilde{\widetilde{a}}: \widetilde{g})=1 / \lambda<+\infty$. In this case the limit superior is the same as that in Theorem 9.6.

\section{Inequalities for the infimal convolution}

The type of a function is often a finer measure of its growth than the order. For this reason we are obliged to study more carefully infimal convolutions of the form $\widetilde{p} \square K$ which appear in Theorem 6.1. This is the aim of the present section and the next.

Throughout this section $E$ denotes a real vector space and $E^{\prime}$ is an arbitrary subspace of its algebraic dual. The Fenchel transform $\widetilde{f}$ is defined on $E^{\prime}$ by (4.1).

Lemma 10.1. Let $f, g: E \rightarrow[-\infty,+\infty]$ be two functions, and assume that $f$ is convex. Then

$$
f \square g(x) \geqslant f(x)-\widetilde{g}(\xi)
$$

for every $x \in E$ such that $f(x)$ is finite and every $\xi \in E^{\prime}$ which is a subgradient of $f$ at the point $x$. In particular, if for every point $x$ there is exactly one subgradient of $f$ in $E^{\prime}$, we may denote it by $f^{\prime}(x)$ and write

$$
f \square g \geqslant f-\widetilde{g} \circ f^{\prime} \quad \text { on } E .
$$

Example. The lemma gives

$$
\left(\frac{1}{6} x^{6}\right) \square\left(\frac{1}{4} x^{4}\right) \geqslant \frac{1}{6} x^{6}-\frac{3}{4} x^{20 / 3}, \quad x \in \mathbf{R},
$$

and, by interchanging $f$ and $g$,

$$
\left(\frac{1}{6} x^{6}\right) \square\left(\frac{1}{4} x^{4}\right) \geqslant \frac{1}{4} x^{4}-\frac{5}{6} x^{18 / 5}, \quad x \in \mathbf{R} .
$$

The first formula gives a good approximation for small $x$, the second for large $x$. To illustrate this we plug in $y=x^{3 / 5}$ in the definition of the infimal convolution, which gives a bound from above:

$$
\left(\frac{1}{6} x^{6}\right) \square\left(\frac{1}{4} x^{4}\right) \leqslant \frac{1}{4} x^{4}-\frac{5}{6} x^{18 / 5}+\frac{3}{2} x^{16 / 5}-x^{14 / 5}+\frac{1}{4} x^{12 / 5}, \quad x \in \mathbf{R} .
$$


Proof of Lemma 10.1. If $A(x)=\xi \cdot x+c$ is an affine function, the convolution with an arbitrary function $g$ is

$$
A \square g=A+C,
$$

where $C$ is the constant $C=-\widetilde{g}(\xi)$. This will give us an inequality for the convolution with a convex function $f$. Let $\xi$ be a subgradient of $f$ at a point $a$ with $-\infty<f(a)<$ $+\infty$, i. e., assume that

$$
f(y) \geqslant f(a)+\xi \cdot(y-a)=A(y), \quad y \in E .
$$

Then the convolution $f \square g$ can be estimated as follows

$$
f \square g(x)=\inf _{y}(f(y) \dot{+} g(x-y)) \geqslant \inf _{y}(A(y)+g(x-y))=A(x)-\widetilde{g}(\xi) .
$$

We have $A(a)=f(a)$ so that for $x=a$,

$$
f \square g(a) \geqslant f(a)-\widetilde{g}(\xi)
$$

When considering the relative type of an entire function we are led to studying the relative type of one infimal convolution $f_{1} \square f_{2}$ with respect to another. Therefore we give below a few results showing that sometimes the type does not change when we convolve with a fixed function.

Lemma 10.2. Let $f_{j}, g_{j}$ be four functions of which the $g_{j}$ are bounded below, $j=1,2$. Then

$$
\operatorname{type}\left(f_{1} \square f_{2}: g_{1} \square g_{2}\right) \leqslant \max _{j=1,2} \operatorname{type}\left(f_{j}: g_{j}\right) \text {. }
$$

If $h$ is bounded below and type $(f: g)=\sigma$, then

$$
\text { type }(f \square(\sigma h): g \square h) \leqslant \sigma .
$$

Proof. For every $b>\operatorname{type}\left(f_{j}: g_{j}\right), j=1,2$, there is a constant $c$ such that $f_{j}(x) \leqslant$ $b g_{j}(x)+c$. Then

$$
f_{1}(y) \dot{+} f_{2}(x-y) \leqslant b\left(g_{1}(y) \dot{+} g_{2}(x-y)\right)+2 c,
$$

and when we take the infimum over all $y$ we get

$$
f_{1} \square f_{2}(x) \leqslant b\left(g_{1} \square g_{2}\right)(x)+2 c ;
$$

hence type $\left(f_{1} \square f_{2}: g_{1} \square g_{2}\right) \leqslant b$. Now $b$ can be taken arbitrarily close to $\max \left(\operatorname{type}\left(f_{j}: g_{j}\right)\right)$.

If $g$ and $h$ are bounded below, the last part of the lemma is a special case of what we have just proved, since type $(\sigma h: h) \leqslant \sigma$. However, on inspecting the proof we see that we need not assume $g$ is bounded below, since now $\sigma h \leqslant b h+C$ for all $b \geqslant \sigma$, not just for $b>\sigma$. 
Lemma 10.3. Let $f_{j}, g_{j}$ be four functions of which $f_{2}$ is bounded below and not identically $+\infty$. Assume that $\widetilde{\widetilde{f}}_{1}=f_{1}, \widetilde{\widetilde{g}}_{1}=g_{1}$, and $\operatorname{dom} \widetilde{f}_{1} \subset \operatorname{dom} \widetilde{f}_{2}$. Then

$$
\operatorname{type}\left(f_{1} \square f_{2}: g_{1} \square g_{2}\right) \geqslant \min \left(\operatorname{type}\left(f_{1}: g_{1}\right), \frac{1}{\operatorname{type}\left(g_{2}: f_{2}\right)}\right) \text {. }
$$

If $f=\widetilde{\widetilde{f}}, g=\widetilde{\widetilde{g}}$ and $h$ are three functions with $h$ bounded below but unbounded above and not equal to $+\infty$ identically, $\sigma=\operatorname{type}(f: g)$, and

$$
\operatorname{dom} \tilde{f} \subset \operatorname{dom}(\sigma h)^{\sim}=\sigma \operatorname{dom} \widetilde{h}
$$

then

$$
\operatorname{type}(f \square(\sigma h): g \square h) \geqslant \sigma \text {. }
$$

Definition 10.4. We shall call the number $1 / \operatorname{type}(g: f)$ the lower type of $f$ relative to $g$.

Proof of Lemma 10.3. If

$$
\operatorname{type}\left(f_{1} \square f_{2}: g_{1} \square g_{2}\right) \geqslant \frac{1}{\operatorname{type}\left(g_{2}: f_{2}\right)}
$$

we have finished. So consider the case when both types are finite and

$$
\operatorname{type}\left(f_{1} \square f_{2}: g_{1} \square g_{2}\right) \cdot \operatorname{type}\left(g_{2}: f_{2}\right)<1 \text {. }
$$

Then there exist numbers $b$ such that

$$
\operatorname{type}\left(f_{1} \square f_{2}: g_{1} \square g_{2}\right)<b \text { and } \operatorname{type}\left(g_{2}: f_{2}\right)<1 / b \text {. }
$$

We claim that for some such $b$ there is a constant $c$ such that

$$
f_{1} \square f_{2} \leqslant b\left(g_{1} \square g_{2}\right)+c \quad \text { and } \quad g_{2} \leqslant \frac{1}{b} f_{2}+c .
$$

In fact, since $f_{2}$ is bounded below, the second inequality holds for all numbers $b<$ $1 / \operatorname{type}\left(g_{2}: f_{2}\right)$, so that $b$ can be chosen arbitrarily close to type $\left(f_{1} \square f_{2}: g_{1} \square g_{2}\right)$.

On taking the Fenchel transformation we get

$$
\widetilde{f}_{1}(\xi)+\widetilde{f}_{2}(\xi) \geqslant b\left(\widetilde{g}_{1}(\xi / b)+\widetilde{g}_{2}(\xi / b)\right)-c
$$

and

$$
b \widetilde{g}_{2}(\xi / b) \geqslant \widetilde{f}_{2}(\xi)-b c
$$

We conclude from (10.5) and (10.6) that

$$
\widetilde{f}_{1}(\xi) \geqslant b \widetilde{g}_{1}(\xi / b)-c-b c .
$$


Indeed, if $\widetilde{f}_{1}(\xi)=+\infty$ or $\widetilde{g}_{1}(\xi / b)=-\infty$ there is nothing to prove, and if not, $\widetilde{f}_{2}(\xi)$ and hence $\widetilde{g}_{2}(\xi / b)$ are finite by hypothesis, and (10.7) follows on adding (10.5) and (10.6). Taking the transform of (10.7) we see that $f_{1} \leqslant b g_{1}+c+b c$. Now let $b$ tend to type $\left(f_{1} \square f_{2}: g_{1} \square g_{2}\right)$. It follows that type $\left(f_{1}: g_{1}\right) \leqslant \operatorname{type}\left(f_{1} \square f_{2}: g_{1} \square g_{2}\right)$.

In the last part of the lemma we have $f_{1}=f, f_{2}=\sigma h, g_{1}=g$ and $g_{2}=h$. So $f_{2}$ is bounded below, and the condition dom $\widetilde{f}_{1} \subset \operatorname{dom} \widetilde{f}_{2}$ is satisfied. Finally $\operatorname{type}\left(g_{2}: f_{2}\right)=\operatorname{type}(h: \sigma h)=1 / \sigma$ if $h$ is unbounded above and not identically $+\infty$.

Example. To illustrate the role of the hypothesis $\operatorname{dom} \widetilde{f}_{1} \subset \operatorname{dom} \widetilde{f}_{2}$ in Lemma 10.3 we shall consider positively homogeneous functions. Given a subset $A$ of $\mathbf{R}^{n}$ we define its supporting function as

$$
H_{A}(\xi)=\sup _{x \in A} \xi \cdot x, \quad \xi \in \mathbf{R}^{n}
$$

This is just the Fenchel transform of the indicator function $i_{A}$ of $A$. Moreover

$$
\left(H_{A} \square H_{B}\right)^{\sim}=\widetilde{H}_{A}+\widetilde{H}_{B}=i_{A}+i_{B}=i_{A \cap B},
$$

if $A$ and $B$ are closed convex sets, so $\left(H_{A} \square H_{B}\right) \approx=H_{A \cap B}$. If in addition one of $A$ and $B$ is bounded, then $H_{A} \square H_{B}$ is Lipschitz continuous, so that

$$
H_{A} \square H_{B}=\left(H_{A} \square H_{B}\right)^{\approx}=H_{A \cap B} .
$$

Now let two compact convex subsets $A_{1}$ and $A_{2}$ be given such that $0 \in A_{1} \cap A_{2}$ and $A_{1}$ is not contained in $A_{2}$. We assume that there exists a number $s<1$ such that $A_{1} \cap A_{2} \subset s A_{2}$ ( $A_{2}$ can for instance be half a ball of sufficiently large radius). We now define $f_{1}=H_{A_{1}}, f_{2}=H_{A_{2}}, g_{1}=g_{2}=s H_{A_{2}}$. Then

$$
f_{1} \square f_{2}=H_{A_{1} \cap A_{2}} \leqslant H_{s A_{2}}=g_{1} \square g_{2},
$$

so that type $\left(f_{1} \square f_{2}: g_{1} \square g_{2}\right) \leqslant 1$. On the other hand, type $\left(f_{1}: g_{1}\right)=\operatorname{type}\left(H_{A_{1}}\right.$ : $\left.s H_{A_{2}}\right)>1$ since $A_{1} \not \subset s A_{2}$, and type $\left(g_{2}: f_{2}\right)=\operatorname{type}\left(s H_{A_{2}}: H_{A_{2}}\right)=s<1$. This shows that (10.4) cannot hold in this case; we have $\operatorname{dom} \tilde{f}_{j}=A_{j}$, so the hypothesis $\operatorname{dom} \widetilde{f}_{1} \subset \operatorname{dom} \widetilde{f}_{2}$ is violated.

Proposition 10.5. Let $f_{j}$ and $g_{j}$ be four functions, all bounded below and not identically $+\infty$, satisfying $\widetilde{\widetilde{f}}_{j}=f_{j}, \widetilde{\widetilde{g}}_{j}=g_{j}$, and $\operatorname{dom} \widetilde{f}_{1}=\operatorname{dom} \widetilde{f}_{2}, \operatorname{dom} \widetilde{g}_{1}=\operatorname{dom} \widetilde{g}_{2}$. Consider the four numbers lower type $\left(f_{j}: g_{j}\right)$, type $\left(f_{j}: g_{j}\right)$, and denote them in increasing order by $\alpha_{1} \leqslant \alpha_{2} \leqslant \alpha_{3} \leqslant \alpha_{4}$. Then

$$
\begin{gathered}
\alpha_{2} \leqslant \operatorname{type}\left(f_{1} \square f_{2}: g_{1} \square g_{2}\right) \leqslant \alpha_{4}, \\
\alpha_{1} \leqslant \text { lower type }\left(f_{1} \square f_{2}: g_{1} \square g_{2}\right) \leqslant \alpha_{3} .
\end{gathered}
$$

If for instance type $\left(f_{1}: g_{1}\right)=\operatorname{type}\left(f_{2}: g_{2}\right)=$ lower type $\left(f_{2}: g_{2}\right)$, then $\operatorname{type}\left(f_{1} \square f_{2}\right.$ : $\left.g_{1} \square g_{2}\right)$ is determined. 
Proof. This is just a combination of (10.3) and (10.4), obtained by interchanging the roles of $f_{j}$ and $g_{j}$.

Example. Let $a_{j k}, b_{j k}$ be positive numbers, $j, k=1,2$, and define $f_{j}(x)=x_{1}^{2} / a_{j 1}+$ $x_{2}^{2} / a_{j 2}$ and $g_{j}(x)=x_{1}^{2} / b_{j 1}+x_{1}^{2} / b_{j 2}$ for $x \in \mathbf{R}^{2}$. Then

$$
\begin{aligned}
f_{1} \square f_{2}(x) & =\frac{x_{1}^{2}}{a_{11}+a_{21}}+\frac{x_{2}^{2}}{a_{12}+a_{22}}, \\
g_{1} \square g_{2}(x) & =\frac{x_{1}^{2}}{b_{11}+b_{21}}+\frac{x_{2}^{2}}{b_{12}+b_{22}} .
\end{aligned}
$$

The types and lower types are, for $j=1,2$,

$$
\begin{gathered}
\sigma_{j}=\operatorname{type}\left(f_{j}: g_{j}\right)=\max _{k}\left(b_{j k} / a_{j k}\right), \quad \tau_{j}=\text { lower type }\left(f_{j}: g_{j}\right)=\min _{k}\left(b_{j k} / a_{j k}\right), \\
\sigma_{3}=\operatorname{type}\left(f_{1} \square f_{2}: g_{1} \square g_{2}\right)=\max _{k}\left(\frac{b_{1 k}+b_{2 k}}{a_{1 k}+a_{2 k}}\right), \\
\tau_{3}=\text { lower type }\left(f_{1} \square f_{2}: g_{1} \square g_{2}\right)=\min _{k}\left(\frac{b_{1 k}+b_{2 k}}{a_{1 k}+a_{2 k}}\right) .
\end{gathered}
$$

By varying the eight numbers $a_{j k}, b_{j k}$ we can see that the inequalities in Proposition 10.5 cannot be improved. In fact, let $\sigma_{j}$ be given and put $a_{j k}=a_{j}, b_{j k}=\sigma_{j} a_{j}$. Then

$$
\sigma_{3}=\max _{k}\left(\frac{b_{1 k}+b_{2 k}}{a_{1 k}+a_{2 k}}\right)=\frac{\sigma_{1} a_{1}+\sigma_{2} a_{2}}{a_{1}+a_{2}}=(1-\lambda) \sigma_{1}+\lambda \sigma_{2}
$$

so that $\sigma_{3}$ can be any number in the segment

$$
] \sigma_{1}, \sigma_{2}\left[=\left\{(1-\lambda) \sigma_{1}+\lambda \sigma_{2} ; 0<\lambda<1\right\} .\right.
$$

Next let $\sigma_{j}$ and $\tau_{j} \leqslant \sigma_{j}$ be given and define $b_{11}=\sigma_{1} a_{11}, b_{12}=\tau_{1} a_{12}, b_{21}=\tau_{2} a_{21}$, $b_{22}=\sigma_{2} a_{22}$. Then

$\sigma_{3}=\max \left(\frac{\sigma_{1} a_{11}+\tau_{2} a_{21}}{a_{11}+a_{21}}, \frac{\tau_{1} a_{12}+\sigma_{2} a_{22}}{a_{12}+a_{22}}\right)=\max \left((1-\lambda) \sigma_{1}+\lambda \tau_{2},(1-\mu) \tau_{1}+\mu \sigma_{2}\right)$,

showing that $\sigma_{3}$ can be the largest of two numbers, one arbitrarily chosen in the segment $] \sigma_{1}, \tau_{2}[$, and the other in the segment $] \tau_{1}, \sigma_{2}\left[\right.$. This proves that $\sigma_{3}$ can be any number in $] \alpha_{2}, \alpha_{4}[$. Similar examples can be constructed in one variable.

\section{Inequalities for the infimal convolution, one variable}

In this section we shall investigate more closely the type of an infimal convolution $f \square h$ with respect to $f$ in the one-dimensional case. We shall assume generally that $f$ and $h$ are defined on the real axis and that $h \geqslant 0, h(t)=+\infty$ when $t>0$; in other words, that $h \geqslant i_{\mathbf{R}_{-}}$. Then, if $f$ is increasing, we will always have $f \square h \geqslant f$ so that

$$
\operatorname{type}(f \square h: f) \geqslant 1 \text { and } \operatorname{type}(f: f \square h) \leqslant 1 \text {. }
$$

In fact,

$$
f \square h(t)=\inf _{s}(f(t-s) \dot{+} h(s)) \geqslant \inf _{s}\left(f(t-s) \dot{+} i_{\mathbf{R}_{-}}(s)\right)=\inf _{s \leqslant 0} f(t-s)=f(t) .
$$

It turns out that equality in the second of the inequalities (11.1) holds under a condition on $h$ which is satisfied by the function $K$ of (6.4), but that equality in the first holds only under a special assumption on $f$. Let us therefore look at the second case first. 
Theorem 11.1. Let $h: \mathbf{R} \rightarrow[0,+\infty]$ be convex and increasing and satisfy $h(t) \rightarrow$ $+\infty$ as $t<0, t \rightarrow 0$. If $\sum h^{-1}\left(c^{j}\right)>-\infty$ for some $c>1$, then $\operatorname{type}(f: f \square h)=1$ for every real-valued increasing function $f$ which is unbounded above.

Proof. We have already remarked that type $(f: f \square h) \leqslant 1$. Suppose that type $(f$ : $f \square h)<1 / c<1$. Then there is some $b<1 / c$ such that $f(t) \leqslant b f(t+s)+h(-s)+C$ for all $t$ and all positive $s$. Repeating this we obtain

$$
f(t) \leqslant b^{k} f\left(t+s_{1}+\cdots+s_{k}\right)+\sum_{1}^{k} b^{j-1}\left(h\left(-s_{j}\right)+C\right) .
$$

Now choose $s_{j}$ such that $h\left(-s_{j}\right)=c^{j-1}$, i.e., $-s_{j}=h^{-1}\left(c^{j-1}\right)$, which implies that $s=\sum_{1}^{\infty} s_{j}=-\sum h^{-1}\left(c^{j-1}\right)<+\infty$. Therefore

$$
f(t) \leqslant b^{k} f(t+s)+\sum_{1}^{\infty}(b c)^{j-1}+\sum_{1}^{\infty} b^{j-1} C=b^{k} f(t+s)+A .
$$

Now let $k \rightarrow+\infty$. If $f$ is real valued we see that $f(t) \leqslant A$, i. e., $f$ is bounded above. If $f$ is both unbounded above and real valued we therefore must have type $(f$ : $f \square h) \geqslant 1 / c$. Now if the hypothesis is satisfied for some $c>1$, it is also satisfied for $\sqrt{c}$. Repeating this we see that the type is at least $c^{-2^{-m}} \rightarrow 1$.

It is easily seen that the function $K$ defined by (6.4) satisfies the condition in the theorem, for $K(-s) \approx-\log s$ when $s>0$ is small, yielding a convergent series with terms $K^{-1}\left(c^{j}\right) \approx-\exp \left(-c^{j}\right)$.

Corollary 11.2. With $f$ and $h$ as in the theorem and $g$ an arbitrary function on $\mathbf{R}$ we have

$$
\begin{aligned}
& \text { lower type }(f: g) \leqslant \text { lower type }(f \square h: g) \leqslant \operatorname{type}(f: g) \leqslant \operatorname{type}(f \square h: g) \text {, } \\
& \text { lower type }(g: f \square h) \leqslant \text { lower } \operatorname{type}(g: f) \leqslant \operatorname{type}(g: f \square h) \leqslant \operatorname{type}(g: f) \text {. }
\end{aligned}
$$

Proof. We only have to observe that

$$
1=\operatorname{type}(f: f \square h) \leqslant \operatorname{type}(f: g) \cdot \operatorname{type}(g: f \square h) .
$$

We now turn to the question of equality in the first inequality of (11.1). This is somewhat more complicated. We first introduce a definition:

Definition 11.3. Let $f, h: \mathbf{R} \rightarrow[-\infty,+\infty]$ be two functions. Assume that both are increasing and that $h \geqslant 0, h(t)=+\infty$ for $t>0$. We shall say that $f$ is $h$-regular for type if type $(f \square h: f)=1$.

Lemma 11.4. Let $f$ and $h$ be two functions as in Definition 11.3. Assume that $\widetilde{\widetilde{f}}=f$ and $\widetilde{\widetilde{h}}=h$. Then the following three conditions are equivalent.

(A) $f$ is h-regular for type.

(B) $f$ is $M$ h-regular for type for every number $M>0$. 
(C) For every $a>1$ there exists a constant $C_{a}$ such that $\tilde{f}(a \tau) / a \geqslant \tilde{f}(\tau)-\widetilde{h}(\tau)-C_{a}$. If in addition $f$ is real valued on some interval $]-\infty, s\left[\right.$ with $s \leqslant+\infty$, and $f^{\prime}(t) \rightarrow+\infty$ as $t<s, t \rightarrow s$, then these conditions are also equivalent to the following (we let $f^{\prime}$ denote a one-sided derivative of $f$ ):

(D) $\widetilde{h}\left(f^{\prime}(t)\right) / f(t) \rightarrow 0$ as $t \rightarrow s$.

We note here that for $h=K$, condition (C) takes the form

$\left(C^{\prime}\right)$ For every $a>1$ there is a constant $C_{a}$ such that

$$
\tilde{f}(a \tau) / a \geqslant \tilde{f}(\tau)+\log \tau-C_{a}, \quad \tau \geqslant 1 .
$$

This is because $-\widetilde{K}(\tau)$ is comparable to $\log \tau$ by (6.5).

Proof. (A) implies (B). We note first that we always have $2 h \leqslant h \square h$. Therefore

$$
\begin{gathered}
f \square(2 h) \leqslant f \square(h \square h)=(f \square h) \square h \leqslant\left(a f+C_{a}\right) \square h \\
\leqslant a(f \square h)+C_{a} \leqslant a\left(a f+C_{a}\right)+C_{a}=a^{2} f+a C_{a}+C_{a}=b f+C_{b}^{\prime} .
\end{gathered}
$$

Here $a$ can come arbitrarily close to 1 , so the same is true of $b=a^{2}$. Thus $f$ is $2 h$-regular for type. It is now obvious how to go on.

(B) implies (C). This follows easily on transforming $f \square(a h) \leqslant a f+C_{a}$.

(C) implies (A). This also follows on taking the transformation.

(A) implies (D). Inequality (10.2) says that

$$
f \square h \geqslant f-\widetilde{h} \circ f^{\prime} .
$$

We just combine this with (A): $f \square h \leqslant a f+C_{a}$ :

$$
0 \leqslant-\widetilde{h}\left(f^{\prime}(t)\right) \leqslant(a-1) f+C_{a} .
$$

We must have $f(t) \rightarrow+\infty$ as $t \rightarrow s$, and we obtain $\lim \sup \left(-\widetilde{h}\left(f^{\prime}\right) / f\right) \leqslant a-1$, a number arbitrarily close to zero.

(D) implies (C). We estimate the convex function $\tilde{f}$ as follows (note that now $\tilde{f}(\tau)$ is real valued for large $\tau)$ :

$$
\frac{1}{a} \widetilde{f}(a \tau)-\widetilde{f}(\tau) \geqslant \frac{1}{a}\left(\widetilde{f}(\tau)+(a \tau-\tau) \widetilde{f}^{\prime}(\tau)\right)-\widetilde{f}(\tau)=\left(1-\frac{1}{a}\right)\left(-\widetilde{f}(\tau)+\tau \widetilde{f}^{\prime}(\tau)\right) .
$$

The last expression is $(1-1 / a) f(t)$ if we choose the right $t$. And (D) means that for $\tau$ large enough, this majorises $-\widetilde{h}\left(f^{\prime}(t)\right)=-\widetilde{h}(\tau)$. Thus (C) holds.

Theorem 11.5. If $f, g: \mathbf{R} \rightarrow[-\infty,+\infty]$ are two increasing functions and one of them is h-regular for type, then

$$
\operatorname{type}(f \square h: g)=\operatorname{type}(f: g) .
$$

Proof. We always have type $(f \square h: g) \geqslant \operatorname{type}(f: g)$, so if $f$ is $h$-regular it follows that

$$
\operatorname{type}(f: g) \leqslant \operatorname{type}(f \square h: g) \leqslant \operatorname{type}(f \square h: f) \cdot \operatorname{type}(f: g) \leqslant \operatorname{type}(f: g),
$$


which implies equality.

If $g$ is $h$-regular we use Lemma 10.2:

$$
\operatorname{type}(f \square h: g \square(h / \sigma)) \leqslant \sigma=\operatorname{type}(f: g),
$$

so

$$
\operatorname{type}(f \square h: g) \leqslant \operatorname{type}(f \square h: g \square(h / \sigma)) \cdot \operatorname{type}(g \square(h / \sigma): g) \leqslant \sigma \text {. }
$$

Theorem 11.6. If $f, g: \mathbf{R} \rightarrow[-\infty,+\infty]$ are two increasing functions with $\widetilde{\widetilde{f}}=f$ and $\widetilde{\widetilde{g}}=g$, and one of them is h-regular for type, then

$$
\operatorname{type}(f: g \square h)=\operatorname{type}(f: g) \text {. }
$$

Proof. We always have type $(f: g \square h) \leqslant \operatorname{type}(f: g)$, so if $g$ is $h$-regular we have finished:

$$
\operatorname{type}(f: g) \leqslant \operatorname{type}(f: g \square h) \cdot \operatorname{type}(g \square h: g) \leqslant \operatorname{type}(f: g \square h) \leqslant \operatorname{type}(f: g) \text {. }
$$

If $f$ is $h$-regular we shall use Lemma 10.3: type $(f \square(\sigma h): g \square h) \geqslant \sigma$. So it follows that

$$
\begin{gathered}
\operatorname{type}(f: g)=\sigma \leqslant \operatorname{type}(f \square(\sigma h): g \square h) \\
\leqslant \operatorname{type}(f \square(\sigma h): f) \cdot \operatorname{type}(f: g \square h)) \leqslant \operatorname{type}(f: g \square h) .
\end{gathered}
$$

Corollary 11.7. If $f$ or $g$ is $h$-regular for type, and $\widetilde{\widetilde{f}}=f, \widetilde{\widetilde{g}}=g$, then

$$
\operatorname{type}(f \square(a h): g \square(b h))=\operatorname{type}(f \square(a h): g)=\operatorname{type}(f: g \square(b h))=\operatorname{type}(f: g)
$$

for all positive numbers $a$ and $b$.

To give an idea about the condition of $h$-regularity we shall now show how to construct functions which do or do not have this property.

It is easy to construct functions that are not $h$-regular. They can be of arbitrarily slow growth, faster than linear growth, and also of arbitrarily fast growth.

Proposition 11.8. Given a positive increasing function $h$ which is plus infinity on the positive axis and satisfies $\widetilde{\widetilde{h}}=h$, and given a function $g: \mathbf{R} \rightarrow \mathbf{R}$ which grows faster than any linear function, there exists a function $f: \mathbf{R} \rightarrow \mathbf{R}$ with $f\left(t_{k}\right)=g\left(t_{k}\right)$ at a sequence which tends to infinity, and which is not h-regular for type.

Proof. We shall define the function $f$ by putting it equal to $g\left(t_{k}\right)$ at the points $t_{k}$ and taking it affine in between. The sequence $t_{k}$ is defined recursively by taking first $t_{0}=0$ and then, if $t_{k}$ is defined, by taking $t_{k+1}$ so large that

$$
\tau_{k}=\frac{g\left(t_{k+1}\right)-g\left(t_{k}\right)}{t_{k+1}-t_{k}}=f^{\prime}\left(t_{k}\right)
$$

satisfies $-\widetilde{h}\left(\tau_{k}\right) \geqslant f\left(t_{k}\right)$. Thus $\lim \sup \left(-\widetilde{h}\left(f^{\prime}(t)\right) / f(t)\right) \geqslant 1$, so that $f$ violates condition (D) of Lemma 11.4.

Depending on a given function $h$, it is sometimes, but not always, possible to construct $h$-regular functions which grow arbitrarily fast. 
Lemma 11.9. If $\varphi$ is h-regular for type and $g \geqslant 0$, then $f=g \square \varphi$ is h-regular for type.

Proof. The regularity condition we are studying is stable under infimal convolution: if we have one function $\varphi$ which satisfies $\varphi \square h \leqslant a \varphi+C_{a}$, then every function $f=g \square \varphi$ with $g \geqslant 0$ does the same:

$$
f \square h=(g \square \varphi) \square h=g \square(\varphi \square h) \leqslant g \square\left(a \varphi+C_{a}\right) \leqslant a(g \square \varphi)+C_{a}=a f+C_{a} .
$$

(This calculation should be compared with the proof that if $\varphi$ is a smooth function, then the usual convolution $f=g * \varphi$ is smooth.)

Proposition 11.10. Assume $h$ satisfies $\widetilde{\widetilde{h}}=h$ and

$$
\int_{1}^{\infty} \frac{|\widetilde{h}(\tau)|}{\tau^{2}} d \tau<+\infty
$$

Then there exist functions $f$ which are h-regular for type, and which grow faster than any given function. But if this condition is not satisfied, the h-regular functions grow at most like the solutions to the differential equation $-\widetilde{h}\left(\varphi^{\prime}\right)=\varphi$, which are real-valued on all of $\mathbf{R}$.

Proof. It is well known that the solutions of an autonomous differential equation $H\left(f^{\prime}\right)=f$ with $H \geqslant 0$ blow up after a finite time if

$$
\int_{1}^{\infty} H(\tau) / \tau^{2} d \tau<+\infty
$$

Translating one such solution $\varphi$ we may assume that it is finite for $\tau<0$ and $=+\infty$ for $\tau>0$. Now if the condition (11.2) is satisfied, there exists a smooth strictly increasing function $H$ such that (11.3) holds and $-\widetilde{h}(\tau)=o(H(\tau))$ as $\tau \rightarrow+\infty$. So the solution $\varphi$ to $H\left(\varphi^{\prime}\right)=\varphi$ satisfies condition (D) of Lemma 11.4. Now let $g \geqslant 0$ be any given function and define $f=g \square\left(\varphi^{+}\right)$. Since $\varphi^{+} \geqslant 0$ and $\varphi=+\infty$ on the positive half-axis we have $f \geqslant g$, and Lemma 11.9 shows that $f$ is $h$-regular for type.

On the other hand it is known that the solutions of the differential equation $H\left(\varphi^{\prime}\right)=\varphi$ are defined over the whole axis if

$$
\int_{1}^{\infty} H(\tau) / \tau^{2} d \tau=+\infty
$$

Any function $f$ which satisfies $-\widetilde{h}\left(f^{\prime}\right) \leqslant f$ has a majorant which is a translate of a fixed solution to $-\widetilde{h}\left(\varphi^{\prime}\right)=\varphi$. Therefore it cannot grow arbitrarily fast if (11.2) is violated.

It should be noted here that $h=K$ satisfies condition (11.2). It is indeed easy to find directly a function $\varphi$ which is infinite on the positive half-axis and $K$-regular for type. We just put $\varphi(t)=-1 / t$ for $t<0, \varphi(t)=+\infty$ for $t \geqslant 0$. Then its Fenchel transform is $\widetilde{\varphi}(\tau)=-2 \sqrt{\tau}$ for $\tau \geqslant 0$ and $\varphi(\tau)=+\infty$ for $\tau \leqslant 0$. So

$$
a^{-1} \widetilde{\varphi}(a \tau)-\widetilde{\varphi}(\tau)=2\left(1-\frac{1}{\sqrt{a}}\right) \sqrt{\tau} \geqslant \log \tau-C_{a}, \quad \tau \geqslant 1,
$$


which means that $\varphi$ satisfies condition $\left(\mathrm{C}^{\prime}\right)$ above. As a consequence, there exist functions which are $K$-regular for type and grow arbitrarily fast, viz. $f=g \square \varphi$ (see Lemma 11.9).

\section{The type of an entire function}

We shall see that knowledge of the function $\widetilde{p}$ defined by the series expansion of an entire function $F$ (see (6.2)) is in general not enough to determine the type. In the positive direction we can prove:

Theorem 12.1. Let $F$ be a nonconstant entire function on $\mathbf{C}^{n}$ and let $f$ be its growth function defined by (6.1), and $p$ its coefficient function defined by (6.2). Then

$$
\operatorname{type}(\widetilde{p}: f)=1 \quad \text { and } \quad \operatorname{type}(f: \widetilde{p}) \geqslant 1 \text {. }
$$

If in addition we assume that $f$ or $\widetilde{p}$ is $K$-regular for type, where $K$ is the function defined by (6.4), then

$$
\operatorname{type}(\widetilde{p}: f)=1 \quad \text { and } \quad \operatorname{type}(f: \widetilde{p})=1 \text {. }
$$

Proof. Cauchy's inequalities $\widetilde{p} \leqslant f$ immediately give type $(\widetilde{p}: f) \leqslant \operatorname{type}(f: f) \leqslant 1$ and type $(f: \widetilde{p}) \geqslant \operatorname{type}(f: f)$. When $f$ is nonconstant, type $(f: f)=1$. Now Theorem 11.1 says that type $(\widetilde{p}: \widetilde{p} \square K)=1$, and this together with the inequality $f \leqslant \widetilde{p} \square K$ (Theorem 6.1) yields type $(\widetilde{p}: f) \geqslant \operatorname{type}(\widetilde{p}: \widetilde{p} \square K)=1$.

In the $K$-regular case we use that type $(f: \widetilde{p})$ is equal to type $(f: \widetilde{p} \square K) \leqslant 1$ (Theorems 11.6 and 6.1). Hence

$$
1=\operatorname{type}(f: f) \leqslant \operatorname{type}(f: \widetilde{p}) \cdot \operatorname{type}(\widetilde{p}: f) \leqslant \operatorname{type}(\widetilde{p}: f) \leqslant 1
$$

which implies equality. (Note that here we do not need to use Theorem 11.1.)

Corollary 12.2. Let $F, G$ be two entire functions defined in $\mathbf{C}^{n}$, let $f, g$ be their growth functions, and $p$ and $q$ their coefficient functions defined by the series expansions (see (6.2)). Assume that one of $f, \widetilde{p}, g, \widetilde{q}$ is $K$-regular for type. Then

$$
\operatorname{type}(F: G)=\operatorname{type}(f: g)=\operatorname{type}(\widetilde{p}: \widetilde{q}) \text {. }
$$

Proof. We have

$$
\widetilde{p} \leqslant f \leqslant \widetilde{p} \square K \leqslant f \square K,
$$

and similarly

$$
\widetilde{q} \leqslant g \leqslant \widetilde{q} \square K \leqslant g \square K .
$$

So everything follows from Corollary 11.7 (in the non-constant case).

The regularity condition of Theorem 12.1 is not always satisfied. We can construct functions such that type $(f: \widetilde{p})>1$.

Example. Consider an entire function $F(z)=\sum a_{j} z^{j}$ with $a_{j}>0$ and such that $p(j)=-\log a_{j}$ is a convex increasing function of $j$. We assume that $p$ is affine on intervals $\left[n_{k}, n_{k+1}\right]$. Then it is easy to see that if these intervals are large enough, 
type $(f: \widetilde{p})$ can be arbitrarily large, even equal to $+\infty$. Let the slope of the coefficient function on the interval $\left[n_{k}, n_{k+1}\right]$ be $t_{k}$, i.e.,

$$
t_{k}=\frac{p\left(n_{k+1}\right)-p\left(n_{k}\right)}{n_{k+1}-n_{k}} .
$$

Then $\widetilde{p}\left(t_{k}\right)=j t_{k}+\log a_{j}$ for $j=n_{k}, \ldots, n_{k+1}$. For $z=e^{t_{k}}$ we get

$$
F(z) \geqslant \sum_{j=n_{k}}^{n_{k+1}} a_{j} e^{j t_{k}}=\left(n_{k+1}-n_{k}+1\right) e^{\tilde{p}\left(t_{k}\right)},
$$

since the terms $a_{j} z^{j}$ are all non-negative when $z \geqslant 0$. This means that

$$
f\left(t_{k}\right) \geqslant \log \left(n_{n+k}-n_{k}+1\right)+\widetilde{p}\left(t_{k}\right) \geqslant \sigma \widetilde{p}\left(t_{k}\right)
$$

for any given positive number $\sigma$ if we only choose $n_{k+1}$ large enough, $t_{k}$ and $n_{k}$ being already chosen. Therefore type $(f: \widetilde{p}) \geqslant \sigma$. We can of course also obtain $f\left(t_{k}\right) \geqslant k \widetilde{p}\left(t_{k}\right)$ so that type $(f: \widetilde{p})=+\infty$. It is clear that this construction works both for slowly growing and fast growing functions.

Example. Let $F$ be the function of the last example, and let $G$ be the lacunary function $G(z)=\sum a_{n_{k}} z^{n_{k}}$; i. e., we retain only the corners on the graph of $p$. Let $g$ be its growth function and $q$ its coefficient function. Then, since $p$ is affine on the intervals $\left[n_{k}, n_{k+1}\right], \widetilde{p}=\widetilde{q}$, so type $(\widetilde{p}: \widetilde{q})=1$. Now for $|z|=e^{t}, t_{k-1} \leqslant t \leqslant t_{k}$, we can estimate $G$ as follows.

$$
\begin{aligned}
|G(z)| & \leqslant a_{n_{k}} e^{n_{k} t}+\sum_{0}^{k-1} a_{n_{j}} e^{n_{j} t}+\sum_{k+1}^{\infty} a_{n_{j}} e^{n_{j} t} \\
& \leqslant a_{n_{k}} e^{n_{k} t}+\frac{a_{n_{k-1}} e^{n_{k-1} t}}{1-\lambda}+\frac{a_{n_{k+1}} e^{n_{k+1} t}}{1-\mu} \leqslant a_{n_{k}} e^{n_{k} t}\left(1+\frac{1}{1-\lambda}+\frac{1}{1-\mu}\right),
\end{aligned}
$$

$\lambda$ and $\mu$ being the ratios of the geometric series whose first two terms agree with that of the respective series; more explicitly we use the simple inequality

$$
\sum_{0}^{\infty} c_{j} \leqslant \sum_{0}^{\infty} c_{0} \lambda^{j}=\frac{c_{0}}{1-\lambda}=\frac{c_{0}}{1-c_{1} / c_{0}}
$$

which holds when $-\log c_{j}$ is convex in $j$. Now

$$
\lambda=\exp \left(-\left(n_{k-1}-n_{k-2}\right)\left(t-t_{k-2}\right)\right) \leqslant \exp \left(-\left(n_{k-1}-n_{k-2}\right)\left(t_{k-1}-t_{k-2}\right)\right)
$$

and

$$
\mu=\exp \left(-\left(n_{k+2}-n_{k+1}\right)\left(t_{k+1}-t\right)\right) \leqslant \exp \left(-\left(n_{k+2}-n_{k+1}\right)\left(t_{k+1}-t_{k}\right)\right)
$$

We can choose $n_{k}$ and $t_{k}$ such that

$$
\left(n_{k}-n_{k-1}\right)\left(t_{k}-t_{k-1}\right) \geqslant \varepsilon>0 \quad \text { and } \quad\left(n_{k+1}-n_{k}\right)\left(t_{k}-t_{k-1}\right) \geqslant \varepsilon>0 .
$$


For example, if the $n_{k}$ are already chosen as in the previous example, then we can choose $t_{k}$, or, if we fix the sequence $\left(t_{k}\right)$ with $t_{k}>t_{k-1}$, then we can choose the $n_{k}$. There is a great freedom in the construction. We now see, writing $C=\left(1-e^{-\varepsilon}\right)^{-1}$, that

$$
|G(z)| \leqslant(2 C+1) a_{n_{k}} e^{n_{k} t}=(2 C+1) e^{\tilde{q}(t)},
$$

so that

$$
g(t) \leqslant \widetilde{q}(t)+\log (2 C+1) .
$$

Thus type $(g: \widetilde{q}) \leqslant 1$. We see finally that the Fenchel transforms of the coefficient functions of the two entire functions $F$ and $G$ are the same: $\widetilde{p}=\widetilde{q}$, but $\operatorname{type}(F$ : $G)=\operatorname{type}(f: g)=\operatorname{type}(f: \widetilde{p})$ can be arbitrarily large. In particular type $(F: G)$ cannot be calculated from $\widetilde{p}, \widetilde{q}$.

This example is in contrast with the classical type which can be determined from $\widetilde{p}$ : it is (for order 1 )

$$
\sigma=\limsup _{t \rightarrow+\infty} f(t) e^{-t}=\limsup _{t \rightarrow+\infty} \widetilde{p}(t) e^{-t} .
$$

This follows from Corollary 12.2 because the exponential function is $K$-regular for type as can easily be shown using, e.g., condition (D) of Lemma 11.4. This fact already shows that the type can be determined from $\widetilde{p}$ in all dimensions, but of course the classical formula

$$
\operatorname{type}(f: \exp )=\limsup \frac{j\left|a_{j}\right|^{\rho / j}}{e \rho}
$$

is more explicit: it does not necessitate calculation of the transform $\widetilde{p}$. 


\section{References}

1. M. Freund and E. GörLich, 'On the relation between maximum modulus, maximum term, and Taylor coefficients of an entire function', J. Approx. Thy 43 (1985) 194-203.

2. W. K. Hayman, 'Note on Hadamard's convexity theorem'. Entire functions and related parts of analysis. Proceedings of Symposia in Pure Mathematics, volume 11, 210-213 (American Mathematical Society, Providence, 1968).

3. O. P. Juneja, G. P. Kapoor and S. K. Bajpai, 'On the $(p, q)$-order and lower $(p, q)$-order of an entire function', J. Reine Angewandte Math. 282 (1976) $53-67$.

4. O. P. Juneja, G. P. Kapoor and S. K. Bajpai, 'On the $(p, q)$-type and lower ( $p, q)$-type of an entire function', J. Reine Angewandte Math. 290 (1977) 180-190.

5. C. O. Kiselman, 'The growth of restrictions of plurisubharmonic functions', Mathematical Analysis and Applications, Part B, L. Nachbin (Ed.). Advances in Mathematics Supplementary Studies, vol. 7B (1981) 435-454.

6. C. O. Kiselman, 'The use of conjugate convex functions in complex analysis', Complex Analysis, J. Ławrynowicz and J. Siciak (Eds.). Banach Center Publications, vol. 11 (1983) 131-142.

7. C. O. Kiselman, 'Croissance des fonctions plurisousharmoniques en dimension infinie', Ann. Inst. Fourier Grenoble 34 (1984) 155-183.

8. C. O. Kiselman, Konvekseco en kompleksa analitiko unu-dimensia (Department of Mathematics, Uppsala University, Lecture Notes 1986:LN2, Uppsala, 1986).

9. B. KJellberG, 'The convexity theorem of Hadamard-Hayman', Proceedings of the symposium in mathematics at the Royal Institute of Technology in June 1973, 87-114 (The Royal Institute of Technology, Stockholm, 1974).

10. P. Lelong and L. Gruman, Entire Functions of Several Variables (SpringerVerlag, 1986).

11. R. T. Rockafellar, Convex Analysis (Princeton University Press, Princeton, 1970).

12. D. SAto, 'On the rate of growth of entire functions of fast growth', Bull. Amer. Math. Soc. 69 (1963) 411-414.

13. J. M. Whittaker, 'The lower order of integral functions', J. London Math. Soc. 8 (1933) 20-27.

Author's address: Uppsala University, Department of Mathematics, P. O. Box 480, SE-751 06 Uppsala, Sweden. 